\title{
Applications of Magnetohydrodynamic Couple Stress Fluid Flow between Two Parallel Plates with Three Different Kernels
}

\author{
Imran Siddique $\mathbb{D}^{1},{ }^{1}$ Ali Akgül, ${ }^{2}$ Hafte Amsalu Kahsay $\mathbb{D}^{3}{ }^{3}$ Teklay Hailay Tsegay, ${ }^{3}$ \\ and Kahsay Godifey Wubneh $\mathbb{D}^{3}$ \\ ${ }^{1}$ Department of Mathematics, University of Management and Technology, Lahore, Pakistan \\ ${ }^{2}$ Department of Mathematics, Siirt University, Art and Science Faculty, TR-56100 Siirt, Turkey \\ ${ }^{3}$ Department of Mathematics, Wollo University, College of Natural Science, Dessie, Ethiopia
}

Correspondence should be addressed to Hafte Amsalu Kahsay; yohanahafte@gmail.com

Received 14 May 2021; Revised 16 July 2021; Accepted 12 October 2021; Published 26 October 2021

Academic Editor: John R. Akeroyd

Copyright (c) 2021 Imran Siddique et al. This is an open access article distributed under the Creative Commons Attribution License, which permits unrestricted use, distribution, and reproduction in any medium, provided the original work is properly cited.

In this paper, we investigate the implementations of newly introduced nonlocal differential operators as convolution of power law, exponential decay law, and the generalized Mittag-Leffler law with fractal derivative in fluid dynamics. The new operators are referred as fractal-fractional differential operators. The governing equations for the problem are constructed with the fractalfractional differential operators. We present the stability analysis and the error analysis.

\section{Introduction}

Magnetohydrodynamics (MHD) deals with the study of the motion of electrically conducting fluids in the presence of the magnetic field. MHD flow has significant importance applications between infinite parallel plates in various areas such as geophysical, astrophysical, and metallurgical processing, MHD generators, pumps, geothermal reservoirs, polymer technology, and mineral industries [1-6]. In last few decades, fractional calculus has taken much interest in many fields $[7,8]$. There are many definitions for the fractional derivative operators, and among them are CaputoFabrizio (CF) [9] and Atangana and Baleanu (AB) [10] definitions of fractional derivatives with a nonlocal and nonsingular kernels having all the characteristics of the old definitions [7, 11-23]. Farman et al. [24] have analyzed the numerical solution of SEIR Epidemic model of measles with noninteger time fractional derivatives by using the Laplace Adomian decomposition method. Ghanbari and Djilali [25] have taken mathematical analysis of a fractional-order predator-prey model with prey social behavior and infection developed in predator population. Ghanbari and Atangana $[26,27]$ have given the new edge detecting techniques based on fractional derivatives with nonlocal and nonsingular kernels. Recently, another idea of differentiation has been proposed by Atanagna [28].

We organize our manuscript as follows. We present the main definitions in Section 2. We construct the problem formulation in Section 3. We present the analysis of the model with the power law kernel in Section 4. We give the analysis of the model with the exponential decay kernel in Section 5. We discuss the analysis of the model with the Mittag-Leffler kernel in Section 6. We present the error analysis in Section 7. We give the conclusion in the last section.

\section{Preminaries}

Definition 1. Assume that $g(\varsigma)$ is a continuous function in the $\left(c_{11}, d_{11}\right)$ and fractal differentiable on $\left(c_{11}, d_{11}\right)$ with order $\eta$ then the fractal-fractional derivative of $g$ of order $\lambda$ in Riemann-Liouville sense with power law kernel is 
introduced as [29]

${ }_{b}^{F F P} D_{t}^{\eta, \lambda} g(\varsigma)=\frac{1}{\Gamma(1-\eta)} \frac{d}{d \varsigma^{\lambda}} \int_{b}^{\varsigma} g(x)(\varsigma-x)^{-\eta} d x, \quad 0<\eta, \lambda \leq 1$,

where

$$
\frac{d g(x)}{d x^{\lambda}}=\lim _{\varsigma \longrightarrow x} \frac{g(\varsigma)-g(x)}{\varsigma^{\lambda}-x^{\lambda}} .
$$

Definition 2. Assume that $g(\varsigma)$ is a continuous function in the $\left(c_{11}, d_{11}\right)$ and fractal differentiable on $\left(c_{11}, d_{11}\right)$ with order $\eta$, then the fractal-fractional derivative of $g$ of order $\lambda$ in Riemann-Liouville sense with the exponential decay kernel is introduced as [29]

$$
{ }_{b}^{F F E} D_{t}^{\eta, \lambda} g(\varsigma)=\frac{M(\eta)}{(1-\eta)} \frac{d}{d \varsigma^{\lambda}} \int_{b}^{\varsigma} g(x) \exp \left(-\frac{\eta}{1-\eta}(\varsigma-x)\right) d x, \quad 0<\eta, \lambda \leq 1 .
$$

Definition 3. Assume that $g(\varsigma)$ is a continuous function in the $\left(c_{11}, d_{11}\right)$ and fractal differentiable on $\left(c_{11}, d_{11}\right)$ with order $\eta$, then the fractal-fractional derivative of $g$ of order $\lambda$ in Riemann-Liouville sense with the generalized MittagLeffler kernel is introduced as [29]

$$
{ }_{b}^{F F E} D_{t}^{\eta, \lambda} g(\varsigma)=\frac{A B(\eta)}{(1-\eta)} \frac{d}{d \varsigma^{\lambda}} \int_{b}^{\varsigma} g(x) E_{\eta}\left(-\frac{\eta}{1-\eta}(\varsigma-x)^{\eta}\right) d x, \quad 0<\eta, \lambda \leq 1 .
$$

\section{Problem Formulation}

We consider

$$
\begin{gathered}
\rho \frac{\partial u_{11}^{\prime}\left(\xi_{11}^{\prime}, \tau_{11}^{\prime}\right)}{\partial \tau_{11}^{\prime}}=\mu \frac{\partial^{2} u_{11}^{\prime}\left(\xi_{11}^{\prime}, \tau_{11}^{\prime}\right)}{\partial \xi_{11}^{\prime 2}}-\eta \frac{\partial^{4} u_{11}^{\prime}\left(\xi_{11}^{\prime}, \tau_{11}^{\prime}\right)}{\partial \xi_{11}^{\prime}}-\sigma B_{0}{ }^{2} u_{11}^{\prime}\left(\xi_{11}^{\prime}, \tau_{11}^{\prime}\right), \\
u_{11}^{\prime}\left(\xi_{11}^{\prime}, 0\right)=0 \text {, for all } \xi_{11}^{\prime}, \quad \text { (initial condition) } \\
u_{11}^{\prime}\left(0, \tau_{11}^{\prime}\right)=0, \quad u_{11}^{\prime}\left(d, \tau_{11}^{\prime}\right)=0, \quad 0 \leq \xi_{11}^{\prime} \leq h, \\
\frac{\partial^{2} u_{11}^{\prime}\left(\xi_{11}^{\prime}, \tau_{11}^{\prime}\right)}{\partial \xi_{11}^{\prime 2}}=0 \text {, at } \xi_{11}^{\prime}=0 \text { and } \xi_{11}^{\prime}=h \text { for any } \tau_{11}^{\prime}>0 \\
v=\frac{u_{11}^{\prime}}{U_{0}}, \quad t=\frac{\tau_{11}^{\prime} U_{0}}{h}, \quad y=\frac{\xi_{11}^{\prime}}{h},
\end{gathered}
$$

into Eqs. (5)-(8), and we obtain

$$
\frac{\partial v(y, t)}{\partial t}=\frac{1}{\operatorname{Re}}\left(\frac{\partial^{2} v(y, t)}{\partial y^{2}}-\frac{\partial^{4} v(y, t)}{\partial y^{4}}-M_{11} v(y, t)\right),
$$

$$
\begin{array}{r}
v(y, 0)=0, \quad v(0, t)=0, \quad v(1, t)=1, \\
\frac{\partial^{2} v(y, t)}{\partial y^{2}}=0, \text { at } y=0 \text { and } y=1 \text { for any } t>0,
\end{array}
$$

where $M_{11}=\sigma B_{0}^{2} d^{2} / \mu$ is the magnetic field parameter, and $\operatorname{Re}=\rho U_{0} d / \mu$ is the Reynold number and $h^{2}=\eta / \mu$. We demonstrate the geometry of the physical model in Figure 1.

\section{Solution of the Problem with the Power Law Kernel}

We take into consideration the Eq. (10) with fractalfractional differential operator using Definition 1 of power law kernel as

$$
{ }_{0}^{F F P} D_{t}^{\alpha_{1}, \beta_{1}} v(y, t)=\frac{1}{\operatorname{Re}}\left(\frac{\partial^{2} v(y, t)}{\partial y^{2}}-\frac{\partial^{4} v(y, t)}{\partial y^{4}}-M_{11} v(y, t)\right),
$$

$$
\begin{aligned}
& \frac{1}{\Gamma\left(1-\alpha_{1}\right)} \frac{d}{d t} \int_{0}^{t} v(y, \lambda)(t-\lambda)^{-\alpha_{1}} d \lambda \\
& \quad=\frac{\beta_{1}}{\operatorname{Re}} t^{\beta_{1}-1}\left(\frac{\partial^{2} v(y, t)}{\partial y^{2}}-\frac{\partial^{4} v(y, t)}{\partial y^{4}}-M_{11} v(y, t)\right) .
\end{aligned}
$$

The, we get

$$
v(y, \lambda)=\frac{\beta_{1}}{\operatorname{Re} \Gamma\left(\alpha_{1}\right)} \int_{0}^{t} \lambda^{\beta_{1}-1}\left(\frac{\partial^{2} v(y, \lambda)}{\partial y^{2}}-\frac{\partial^{4} v(y, \lambda)}{\partial y^{4}}-M_{11} v(y, \lambda)\right)(t-\lambda)^{\alpha_{1}-1} d \lambda .
$$

For simplicity, we take

$$
\begin{gathered}
F(y, \lambda)=\beta_{1} \lambda^{\beta_{1}-1}\left(\frac{\partial^{2} v(y, \lambda)}{\partial y^{2}}-\frac{\partial^{4} v(y, \lambda)}{\partial y^{4}}-M_{11} v(y, \lambda)\right), \\
v(y, \lambda)=\frac{1}{\operatorname{Re} \Gamma\left(\alpha_{1}\right)} \int_{0}^{t} F(y, \lambda)(t-\lambda)^{\alpha_{1}-1} d \lambda .
\end{gathered}
$$

We discretize this equation at $\left(y_{i}, t=t_{n+1}\right)$ and get

$$
\begin{gathered}
v\left(y_{i}, t_{n+1}\right)=\frac{1}{\operatorname{Re} \Gamma\left(\alpha_{1}\right)} \int_{0}^{t_{n+1}} F\left(y_{i}, \lambda\right)\left(t_{n+1}-\lambda\right)^{\alpha_{1}-1} d \lambda, \\
v\left(y_{i}, t_{n+1}\right)=\frac{1}{\operatorname{Re} \Gamma\left(\alpha_{1}\right)} \sum_{j=0}^{n} \int_{t_{j}}^{t_{j+1}} F\left(y_{i}, \lambda\right)\left(t_{n+1}-\lambda\right)^{\alpha_{1}-1} d \lambda .
\end{gathered}
$$

We apply the two-step Lagrange polynomial as

$$
p_{j}(\lambda)=\frac{\lambda-t_{j-1}}{t_{j}-t_{j-1}} F\left(y_{i}, t_{j}\right)-\frac{\lambda-t_{j}}{t_{j}-t_{j-1}} F\left(y_{i}, t_{j-1}\right) .
$$


Thus, we will get

$$
\begin{aligned}
v\left(y_{i}, t_{n+1}\right)= & \frac{1}{\operatorname{Re} \Gamma\left(\alpha_{1}\right)} \sum_{j=0}^{n} \int_{t_{j}}^{t_{j+1}} p_{j}(\lambda)\left(t_{n+1}-\lambda\right)^{\alpha_{1}-1} d \lambda \\
= & \sum_{j=0}^{n}\left[\frac { h ^ { \alpha _ { 1 } } F ( y _ { i } , t _ { j } ) } { \operatorname { R e } \Gamma ( \alpha _ { 1 } + 2 ) } \left((n+1-j)^{\alpha_{1}}\left(n-j+2+\alpha_{1}\right)\right.\right. \\
& \left.\left.-(n-j)^{\alpha_{1}}\left(n-j+2+2 \alpha_{1}\right)\right)\right] \\
& -\sum_{j=0}^{n}\left[\frac{h^{\alpha_{1}} F\left(y_{i}, t_{j-1}\right)}{\operatorname{Re} \Gamma\left(\alpha_{1}+2\right)}\left((n+1-j)^{\alpha_{1}+1}-(n-j)^{\alpha_{1}}\left(n-j+1+\alpha_{1}\right)\right)\right] .
\end{aligned}
$$

We have

$$
F\left(y_{i}, t_{j}\right)=\beta_{1} t_{j}^{\beta_{1}-1}\left(\frac{v_{i+1}^{j}-2 v_{i}^{j}+v_{i-1}^{j}}{(\Delta y)^{2}}-\frac{v_{i+2}^{j}-4 v_{i+1}^{j}+6 v_{i}^{j}-4 v_{i-1}^{j}+v_{i-2}^{j}}{(\Delta y)^{4}}-M_{11} v_{i}^{j}\right) .
$$

Then, we will obtain

$$
\begin{aligned}
v\left(y_{i}, t_{n+1}\right)= & \frac{1}{\operatorname{Re} \Gamma\left(\alpha_{1}\right)} \sum_{j=0}^{n} \int_{t_{j}}^{t_{j+1}} p_{j}(\lambda)\left(t_{n+1}-\lambda\right)^{\alpha_{1}-1} d \lambda \\
= & \sum_{j=0}^{n}\left[\frac{h^{\alpha_{1}} \beta_{1} t_{j}^{\beta_{1}-1}}{\operatorname{Re} \Gamma\left(\alpha_{1}+2\right)}\right. \\
& \cdot\left(\frac{v_{i+1}^{j}-2 v_{i}^{j}+v_{i-1}^{j}}{(\Delta y)^{2}}-\frac{v_{i+2}^{j}-4 v_{i+1}^{j}+6 v_{i}^{j}-4 v_{i-1}^{j}+v_{i-2}^{j}}{(\Delta y)^{4}}-M_{11} v_{i}^{j}\right) \\
= & \left.\times\left((n+1-j)^{\alpha_{1}}\left(n-j+2+\alpha_{1}\right)-(n-j)^{\alpha_{1}}\left(n-j+2+2 \alpha_{1}\right)\right)\right] \\
& -\sum_{j=0}^{n}\left[\frac{h^{\alpha_{1}} \beta_{1} t_{j-1}^{\beta_{1}-1}}{\operatorname{Re} \Gamma\left(\alpha_{1}+2\right)}\right. \\
& \cdot\left(\frac{v_{i+1}^{j-1}-2 v_{i}^{j-1}+v_{i-1}^{j-1}}{(\Delta y)^{2}}-\frac{v_{i+2}^{j-1}-4 v_{i+1}^{j-1}+6 v_{i}^{j-1}-4 v_{i-1}^{j-1}+v_{i-2}^{j-1}}{(\Delta y)^{4}}-M_{11} v_{i}^{j-1}\right) \\
& \left.\times\left((n+1-j)^{\alpha_{1}+1}-(n-j)^{\alpha_{1}}\left(n-j+1+\alpha_{1}\right)\right)\right] .
\end{aligned}
$$

We define

$$
\begin{gathered}
A_{n, j}^{\alpha_{1}, \beta_{1}}=\frac{h^{\alpha_{1}} \beta_{1} t_{j}^{\beta_{1}-1} K_{n, j}^{\alpha_{1}}}{\operatorname{Re} \Gamma\left(\alpha_{1}+2\right)(\Delta y)^{2}}, \quad B_{n, j}^{\alpha_{1}, \beta_{1}}=\frac{h^{\alpha_{1}} \beta_{1} t_{j}^{\beta_{1}-1} K_{n, j}^{\alpha_{1}}}{\operatorname{Re} \Gamma\left(\alpha_{1}+2\right)(\Delta y)^{4}}, \\
C_{n, j}^{\alpha_{1}, \beta_{1}}=\frac{h^{\alpha_{1}} \beta_{1} t_{j}^{\beta_{1}-1} M_{11} K_{n, j}^{\alpha_{1}}}{\operatorname{Re} \Gamma\left(\alpha_{1}+2\right)}, \\
D_{n, j-1}^{\alpha_{1}, \beta_{1}}=\frac{h^{\alpha_{1}} \beta_{1} t_{j-1}^{\beta_{1}-1} \gamma_{n, j}^{\alpha_{1}}}{\operatorname{Re} \Gamma\left(\alpha_{1}+2\right)(\Delta y)^{2}}, \quad B_{n, j-1}^{\alpha_{1}, \beta_{1}}=\frac{h^{\alpha_{1}} \beta_{1} t_{j-1}^{\beta_{1}-1} \gamma_{n, j}^{\alpha_{1}}}{\operatorname{Re} \Gamma\left(\alpha_{1}+2\right)(\Delta y)^{4}}, \\
C_{n, j-1}^{\alpha_{1}, \beta_{1}}=\frac{h^{\alpha_{1}} \beta_{1} t_{j}^{\beta_{1}-1} M_{11} \gamma_{n, j}^{\alpha_{1}}}{\operatorname{Re} \Gamma\left(\alpha_{1}+2\right)},
\end{gathered}
$$

$$
K_{n, j}^{\alpha_{1}}=\left((n+1-j)^{\alpha_{1}}\left(n-j+2+\alpha_{1}\right)-(n-j)^{\alpha_{1}}\left(n-j+2+2 \alpha_{1}\right)\right),
$$$$
\gamma_{n, j}^{\alpha_{1}}=\left((n+1-j)^{\alpha_{1}+1}-(n-j)^{\alpha_{1}}\left(n-j+1+\alpha_{1}\right)\right) .
$$

Then, we get

$$
\begin{aligned}
v_{i}^{n+1}= & \sum_{j=0}^{n}\left[A_{n, j}^{\alpha_{1}, \beta_{1}}\left(v_{i+1}^{j}-2 v_{i}^{j}+v_{i-1}^{j}\right)-B_{n, j}^{\alpha_{1}, \beta_{1}}\right. \\
& \left.\cdot\left(v_{i+2}^{j}-4 v_{i+1}^{j}+6 v_{i}^{j}-4 v_{i-1}^{j}+v_{i-2}^{j}\right)-C_{n, j}^{\alpha_{1}, \beta_{1}} v_{i}^{j}\right] \\
& -\sum_{j=0}^{n}\left[D_{n, j-1}^{\alpha_{1}, \beta_{1}}\left(v_{i+1}^{j-1}-2 v_{i}^{j-1}+v_{i-1}^{j-1}\right)\right. \\
& \left.-E_{n, j-1}^{\alpha_{1}, \beta_{1}}\left(v_{i+2}^{j-1}-4 v_{i+1}^{j-1}+6 v_{i}^{j-1}-4 v_{i-1}^{j-1}+v_{i-2}^{j-1}\right)-F_{n, j-1}^{\alpha_{1}, \beta_{1}}\left(v_{i}^{j-1}\right)\right] .
\end{aligned}
$$

We choose $\varepsilon_{i}^{n}=\delta_{n} \exp \left(i k_{m} y\right)$. Then, we have

$$
\begin{aligned}
& \delta_{n+1} \exp \left(i k_{m} y\right)=A_{n, n}^{\alpha_{1}, \beta_{1}}\left[\delta_{n} \exp \left(i k_{m}(y+\Delta y)\right)-2 \delta_{n} \exp \left(i k_{m} y\right)+\delta_{n} \exp \left(i k_{m}(y-\Delta y)\right)\right] \\
& -B_{n, n}^{\alpha_{1} \beta_{1}}\left(\begin{array}{c}
\delta_{n} \exp \left(i k_{m}(y+2 \Delta y)\right)-4 \delta_{n} \exp \left(i k_{m}(y+\Delta y)\right)+6 \delta_{n} \exp \left(i k_{m} y\right) \\
-4 \delta_{n} \exp \left(i k_{m}(y-\Delta y)\right)+\delta_{n} \exp \left(i k_{m}(y-2 \Delta y)\right)
\end{array}\right) \\
& -C_{n, n}^{\alpha_{1}, \beta_{1}} \delta_{n} \exp \left(i k_{m} y\right)-D_{n, n-1}^{\alpha_{1}, \beta_{1}}\left[\delta_{n-1} \exp \left(i k_{m}(y+\Delta y)\right)-2 \delta_{n-1} \exp \left(i k_{m} y\right)\right. \\
& \left.+\delta_{n-1} \exp \left(i k_{m}(y-\Delta y)\right)\right] \\
& -E_{n, n-1}^{\alpha_{1}, \beta_{1}}\left(\begin{array}{c}
\delta_{n-1} \exp \left(i k_{m}(y+2 \Delta y)\right)-4 \delta_{n-1} \exp \left(i k_{m}(y+\Delta y)\right)+6 \delta_{n-1} \exp \left(i k_{m} y\right) \\
-4 \delta_{n-1} \exp \left(i k_{m}(y-\Delta y)\right)+\delta_{n-1} \exp \left(i k_{m}(y-2 \Delta y)\right)
\end{array}\right) \\
& -F_{n, n-1}^{\alpha_{1}, \beta_{1}}\left(\delta_{n-1} \exp \left(i k_{m} y\right)\right)
\end{aligned}
$$

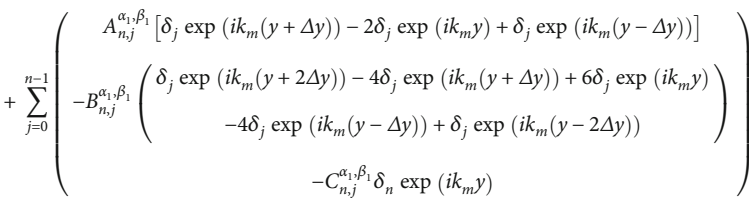

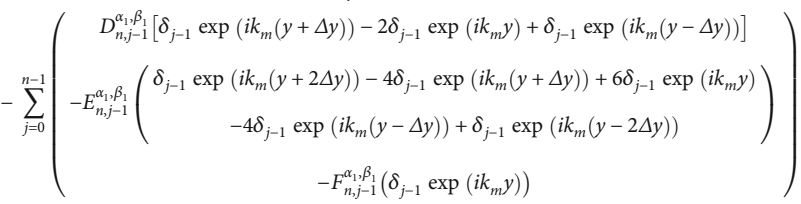

\section{After simplification, we obtain}

$$
\begin{aligned}
& \delta_{n+1}=A_{n, n}^{\alpha_{1}, \beta_{1}}\left[\delta_{n} \exp \left(i k_{m} \Delta y\right)-2 \delta_{n}+\delta_{n} \exp \left(-i k_{m} \Delta y\right)\right]-B_{n, n}^{\alpha_{1}, \beta_{1}} \\
& \cdot\left(\begin{array}{c}
\delta_{n} \exp \left(2 i k_{m} \Delta y\right)-4 \delta_{n} \exp \left(i k_{m} \Delta y\right)+6 \delta_{n} \\
-4 \delta_{n} \exp \left(i k_{m} \Delta y\right)+\delta_{n} \exp \left(-2 i k_{m} \Delta y\right)
\end{array}\right) \\
& -C_{n, n}^{\alpha_{1}, \beta_{1}} \delta_{n}-D_{n, n-1}^{\alpha_{1}, \beta_{1}}\left[\delta_{n-1} \exp \left(i k_{m} \Delta y\right)-2 \delta_{n-1}+\delta_{n-1} \exp \left(i k_{m} \Delta y\right)\right]-E_{n, n-1}^{\alpha_{1}, \beta_{1}} \\
& \cdot\left(\begin{array}{c}
\delta_{n-1} \exp \left(2 i k_{m} \Delta y\right)-4 \delta_{n-1} \exp \left(i k_{m} \Delta y\right)+6 \delta_{n-1} \\
-4 \delta_{n-1} \exp \left(i k_{m} \Delta y\right)+\delta_{n-1} \exp \left(-2 i k_{m} \Delta y\right)
\end{array}\right)-F_{n, n-1}^{\alpha_{1}, \beta_{1}} \delta_{n-1}+\sum_{j=0}^{n-1} \\
& \left(\begin{array}{c}
A_{n, j}^{\alpha_{1}, \beta_{1}}\left[\delta_{j} \exp \left(i k_{m} \Delta y\right)-2 \delta_{j}+\delta_{j} \exp \left(-i k_{m} \Delta y\right)\right] \\
-B_{n, j}^{\alpha_{1}, \beta_{1}}\left(\begin{array}{c}
\delta_{j} \exp \left(2 i k_{m} \Delta y\right)-4 \delta_{j} \exp \left(i k_{m} \Delta y\right)+6 \delta_{j} \\
-4 \delta_{j} \exp \left(-i k_{m} \Delta y\right)+\delta_{j} \exp -2\left(i k_{m} \Delta y\right)
\end{array}\right) \\
-C_{n, j}^{\alpha_{1}, \beta_{1}} \delta_{n}
\end{array}\right) \\
& -\sum_{j=0}^{n-1}\left(\begin{array}{c}
D_{n, j-1}^{\alpha_{1}, \beta_{1}}\left[\delta_{j-1} \exp \left(i k_{m} \Delta y\right)-2 \delta_{j-1}+\delta_{j-1} \exp \left(-i k_{m} \Delta y\right)\right] \\
-E_{n, j-1}^{\alpha_{1}, \beta_{1}}\left(\begin{array}{c}
\delta_{j-1} \exp \left(2 i k_{m} \Delta y\right)-4 \delta_{j-1} \exp \left(i k_{m} \Delta y\right)+6 \delta_{j-1} \\
-4 \delta_{j-1} \exp \left(-i k_{m} \Delta y\right)+\delta_{j-1} \exp \left(-2 i k_{m} \Delta y\right)
\end{array}\right) \\
-F_{n, j-1}^{\alpha_{1}, \beta_{1}} \delta_{j-1}
\end{array}\right) .
\end{aligned}
$$




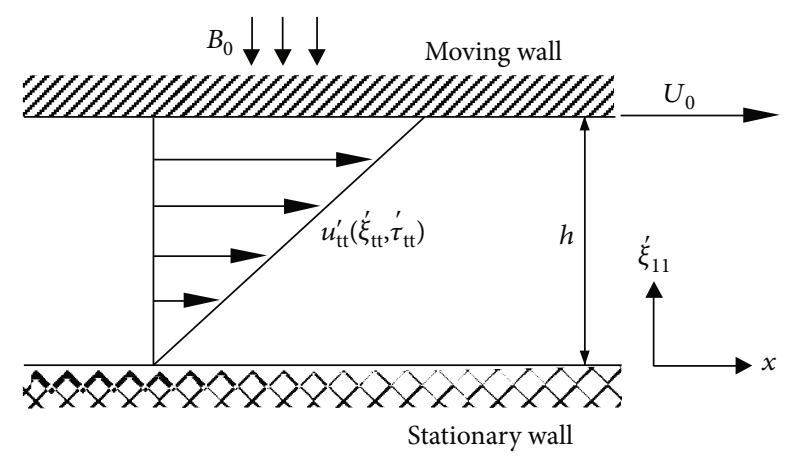

Figure 1: Geometry of the physical model.

We prove by induction. For $n=0$, we obtain

$\delta_{1}=A_{0,0}^{\alpha_{1}, \beta_{1}}\left[\delta_{0} \exp \left(i k_{m} \Delta y\right)-2 \delta_{0}+\delta_{0} \exp \left(-i k_{m} \Delta y\right)\right]-B_{0,0}^{\alpha_{1}, \beta_{1}}$

$\cdot\left(\begin{array}{c}\delta_{0} \exp \left(2 i k_{m} \Delta y\right)-4 \delta_{0} \exp \left(i k_{m} \Delta y\right)+6 \delta_{n} \\ -4 \delta_{0} \exp \left(i k_{m} \Delta y\right)+\delta_{0} \exp \left(-2 i k_{m} \Delta y\right)\end{array}\right)-C_{0,0}^{\alpha_{1}, \beta_{1}} \delta_{0}$

$=\delta_{0}\left(\begin{array}{c}A_{0,0}^{\alpha_{1}, \beta_{1}}\left[\exp \left(i k_{m} \Delta y\right)-2+\exp \left(-i k_{m} \Delta y\right)\right] \\ -B_{0,0}^{\alpha_{1}, \beta_{1}}\left(\begin{array}{c}\exp \left(2 i k_{m} \Delta y\right)-4 \exp \left(i k_{m} \Delta y\right)+6 \delta_{n} \\ -4 \exp \left(i k_{m} \Delta y\right)+\exp \left(-2 i k_{m} \Delta y\right)\end{array}\right)-C_{0,0}^{\alpha_{1}, \beta_{1}}\end{array}\right)$

$=\delta_{0}\left(-4 \sin ^{2}\left(\frac{k_{m} \Delta y}{2}\right) A_{0,0}^{\alpha_{1}, \beta_{1}}+4 B_{0,0}^{\alpha_{1}, \beta_{1}}\left(\sin ^{2}\left(k_{m} \Delta y\right)+4 \sin ^{2}\left(\frac{k_{m} \Delta y}{2}\right)\right)-C_{0,0}^{\alpha_{1}, \beta_{1}}\right)$.

We should show $\left|\delta_{1} / \delta_{0}\right|<1$. Therefore, we have

$\left|-4 \sin ^{2}\left(\frac{k_{m} \Delta y}{2}\right) A_{0,0}^{\alpha_{1}, \beta_{1}}+4 B_{0,0}^{\alpha_{1}, \beta_{1}}\left(\sin ^{2}\left(k_{m} \Delta y\right)+4 \sin ^{2}\left(\frac{k_{m} \Delta y}{2}\right)\right)-C_{0,0}^{\alpha_{1}, \beta_{1}}\right|<1 . "$

Since we have for all $m$, we obtain

$$
"\left|-4 A_{0,0}^{\alpha_{1}, \beta_{1}}+20 B_{0,0}^{\alpha_{1}, \beta_{1}}-C_{0,0}^{\alpha_{1}, \beta_{1}}\right|<1 .
$$

When $n>1$, we have

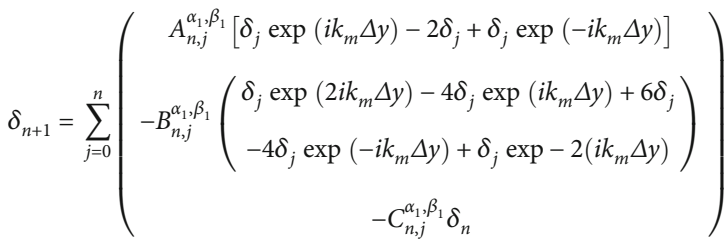

$$
\begin{aligned}
& -\sum_{j=0}^{n-1}\left(\begin{array}{c}
D_{n, j-1}^{\alpha_{1}, \beta_{1}}\left[\delta_{j-1} \exp \left(i k_{m} \Delta y\right)-2 \delta_{j-1}+\delta_{j-1} \exp \left(-i k_{m} \Delta y\right)\right] \\
-E_{n, j-1}^{\alpha_{1}, \beta_{1}}\left(\begin{array}{c}
\delta_{j-1} \exp \left(2 i k_{m} \Delta y\right)-4 \delta_{j-1} \exp \left(i k_{m} \Delta y\right)+6 \delta_{j-1} \\
-4 \delta_{j-1} \exp \left(-i k_{m} \Delta y\right)+\delta_{j-1} \exp \left(-2 i k_{m} \Delta y\right)
\end{array}\right) \\
-F_{n, j-1}^{\alpha_{1}, \beta_{1}} \delta_{j-1}
\end{array}\right)
\end{aligned}
$$

Then, we get

$$
\begin{aligned}
\delta_{n+1}= & \sum_{j=0}^{n} \delta_{j}\left(-4 \sin ^{2}\left(\frac{k_{m} \Delta y}{2}\right) A_{n, j}^{\alpha_{1}, \beta_{1}}+4 B_{n, j}^{\alpha_{1}, \beta_{1}}\right. \\
& \left.\cdot\left(\sin ^{2}\left(k_{m} \Delta y\right)+4 \sin ^{2}\left(\frac{k_{m} \Delta y}{2}\right)\right)-C_{n, j}^{\alpha_{1}, \beta_{1}}\right) \\
& -\sum_{j=0}^{n-1} \delta_{j-1}\left(-4 \sin ^{2}\left(\frac{k_{m} \Delta y}{2}\right) A_{n, j-1}^{\alpha_{1}, \beta_{1}}+4 B_{n, j-1}^{\alpha_{1}, \beta_{1}}\right. \\
& \left.\cdot\left(\sin ^{2}\left(k_{m} \Delta y\right)+4 \sin ^{2}\left(\frac{k_{m} \Delta y}{2}\right)\right)-C_{n, j-1}^{\alpha_{1}, \beta_{1}}\right) .
\end{aligned}
$$

We assume that for all $n \geq 1,\left|\delta_{1} / \delta_{0}\right|<1$. We want to prove that $\left|\delta_{n+1} / \delta_{0}\right|<1$.. However,

$$
\begin{aligned}
\left|\delta_{n+1}\right| \leq & \sum_{j=0}^{n}\left|\delta_{j}\right| \mid-4 \sin ^{2}\left(\frac{k_{m} \Delta y}{2}\right) A_{n, j}^{\alpha_{1}, \beta_{1}}+4 B_{n, j}^{\alpha_{1}, \beta_{1}} \\
& \cdot\left(\sin ^{2}\left(k_{m} \Delta y\right)+4 \sin ^{2}\left(\frac{k_{m} \Delta y}{2}\right)\right) \\
& -C_{n, j}^{\alpha_{1}, \beta_{1}}\left|+\sum_{j=0}^{n-1}\right| \delta_{j-1}||-4 \sin ^{2}\left(\frac{k_{m} \Delta y}{2}\right) A_{n, j-1}^{\alpha_{1}, \beta_{1}}+4 B_{n, j-1}^{\alpha_{1}, \beta_{1}} \\
& \cdot\left(\sin ^{2}\left(k_{m} \Delta y\right)+4 \sin ^{2}\left(\frac{k_{m} \Delta y}{2}\right)\right)-C_{n, j-1}^{\alpha_{1}, \beta_{1}} \mid "
\end{aligned}
$$

By induction hypothesis for all $n \geq 1,\left|\delta_{n}\right|<\left|\delta_{0}\right|$, we have

$$
\begin{aligned}
\left|\delta_{n+1}\right|< & \left|\delta_{0}\right|\left(\sum_{j=0}^{n} \mid\left(-4 \sin ^{2}\left(\frac{k_{m} \Delta y}{2}\right) A_{n, j}^{\alpha_{1}, \beta_{1}}+4 B_{n, j}^{\alpha_{1}, \beta_{1}}\right.\right. \\
& \left.\cdot\left(\sin ^{2}\left(k_{m} \Delta y\right)+4 \sin ^{2}\left(\frac{k_{m} \Delta y}{2}\right)\right)-C_{n, j}^{\alpha_{1}, \beta_{1}}\right)\left|+\sum_{j=0}^{n-1}\right| \\
& \cdot\left(-4 \sin ^{2}\left(\frac{k_{m} \Delta y}{2}\right) A_{n, j-1}^{\alpha_{1}, \beta_{1}}+4 B_{n, j-1}^{\alpha_{1}, \beta_{1}}\right. \\
& \left.\left.\cdot\left(\sin ^{2}\left(k_{m} \Delta y\right)+4 \sin ^{2}\left(\frac{k_{m} \Delta y}{2}\right)\right)-C_{n, j-1}^{\alpha_{1}, \beta_{1}}\right) \mid\right) .
\end{aligned}
$$

This inequality is true for all $m$. Thus, we reach

$$
\begin{aligned}
&\left|\delta_{n+1}\right|<\left|\delta_{0}\right|\left(\sum_{j=0}^{n}\left|\left(-4 A_{n, j}^{\alpha_{1}, \beta_{1}}+20 B_{n, j}^{\alpha_{1}, \beta_{1}}-C_{n, j}^{\alpha_{1}, \beta_{1}}\right)\right|+\sum_{j=0}^{n-1} \mid\right. \\
&\left.\cdot\left(-4 A_{n, j-1}^{\alpha_{1}, \beta_{1}}+20 B_{n, j-1}^{\alpha_{1}, \beta_{1}}-C_{n, j-1}^{\alpha_{1}, \beta_{1}}\right) \mid\right) .
\end{aligned}
$$

We need to show that $\left|\delta_{n+1} / \delta_{0}\right|<1$. Thus, we reach

$$
\left(\sum_{j=0}^{n}\left|\left(-4 A_{n, j}^{\alpha_{1}, \beta_{1}}+20 B_{n, j}^{\alpha_{1}, \beta_{1}}-C_{n, j}^{\alpha_{1}, \beta_{1}}\right)\right|+\sum_{j=0}^{n-1}\left|\left(-4 A_{n, j-1}^{\alpha_{1}, \beta_{1}}+20 B_{n, j-1}^{\alpha_{1}, \beta_{1}}-C_{n, j-1}^{\alpha_{1}, \beta_{1}}\right)\right|\right)<1 .
$$




\section{Solution of the Problem with the Exponential Decay Kernel}

We consider Eq. (10) with fractal-fractional differential operator using Definition 2 of exponential decay kernel as

$$
\begin{gathered}
{ }_{0}^{F F E} D_{t}^{\alpha_{1}, \beta_{1}} v(y, t)=\frac{1}{\operatorname{Re}}\left(\frac{\partial^{2} v(y, t)}{\partial y^{2}}-\frac{\partial^{4} v(y, t)}{\partial y^{4}}-M_{11} v(y, t)\right) \\
\frac{M\left(\alpha_{1}\right)}{\left(1-\alpha_{1}\right)} \frac{d}{d t} \int_{0}^{t} v(y, \lambda) \exp \left(-\frac{\alpha_{1}}{\left(1-\alpha_{1}\right)}(t-\lambda)\right) d \lambda \\
=\frac{\beta_{1}}{\operatorname{Re}} t^{\beta_{1}-1}\left(\frac{\partial^{2} v(y, t)}{\partial y^{2}}-\frac{\partial^{4} v(y, t)}{\partial y^{4}}-M_{11} v(y, t)\right)
\end{gathered}
$$

For simplicity, we define

$$
F(y, t, v(y, t))=\frac{\beta_{1}}{\operatorname{Re}} t^{\beta_{1}-1}\left(\frac{\partial^{2} v(y, t)}{\partial y^{2}}-\frac{\partial^{4} v(y, t)}{\partial y^{4}}-M_{11} v(y, t)\right) .
$$

Then, we reach

$$
v(y, \lambda)=\frac{1-\alpha_{1}}{M\left(\alpha_{1}\right)} F(y, t, v(y, t))+\frac{\alpha_{1}}{M\left(\alpha_{1}\right)} \int_{0}^{t} F(y, \lambda, v(y, \lambda)) d \lambda .
$$

We discretize Eq. $(36)$ at $\left(y_{i}, t_{n+1}\right)$ and $\left(y_{i}, t_{n}\right)$ as

$$
\begin{gathered}
v_{i}^{n+1}=\frac{1-\alpha_{1}}{M\left(\alpha_{1}\right)} F\left(y_{i}, t_{n}, v_{i}^{n}\right)+\frac{\alpha_{1}}{M\left(\alpha_{1}\right)} \int_{0}^{t_{n+1}} F\left(y_{i}, \lambda, v\left(y_{i}, \lambda\right)\right) d \lambda, \\
v_{i}^{n}=\frac{1-\alpha_{1}}{M\left(\alpha_{1}\right)} F\left(y_{i}, t_{n}, v_{i}^{n-1}\right)+\frac{\alpha_{1}}{M\left(\alpha_{1}\right)} \int_{0}^{t_{n}} F\left(y_{i}, \lambda, v\left(y_{i}, \lambda\right)\right) d \lambda .
\end{gathered}
$$

Then, we obtain

$$
\begin{aligned}
v_{i}^{n+1}= & v_{i}^{n}+\frac{1-\alpha_{1}}{M\left(\alpha_{1}\right)}\left(F\left(y_{i}, t_{n}, v_{i}^{n}\right)-F\left(y_{i}, t_{n-1}, v_{i}^{n-1}\right)\right) \\
& +\frac{\alpha_{1}}{M\left(\alpha_{1}\right)} \int_{t_{n}}^{t_{n+1}} F\left(y_{i}, \lambda, v\left(y_{i}, \lambda\right)\right) d \lambda \\
= & v_{i}^{n}+\frac{1-\alpha_{1}}{M\left(\alpha_{1}\right)}\left(F\left(y_{i}, t_{n}, v_{i}^{n}\right)-F\left(y_{i}, t_{n-1}, v_{i}^{n-1}\right)\right) \\
& +\frac{\alpha_{1}}{M\left(\alpha_{1}\right)}\left(\frac{3 h}{2} F\left(y_{i}, t_{n}, v_{i}^{n}\right)-\frac{h}{2} F\left(y_{i}, t_{n-1}, v_{i}^{n-1}\right)\right),
\end{aligned}
$$

where

$$
\begin{aligned}
& F\left(y_{i}, t_{n}, v\left(y_{i}, t_{n}\right)\right) \\
& \quad=\frac{\beta_{1}}{\operatorname{Re}} t_{n}^{\beta_{1}-1}\left(\frac{v_{i+1}^{n}-2 v_{i}^{n}+v_{i-1}^{n}}{(\Delta y)^{2}}-\frac{v_{i+2}^{n}-4 v_{i+1}^{n}+6 v_{i}^{n}-4 v_{i-1}^{n}+v_{i-2}^{n}}{(\Delta y)^{4}}-M_{11} v_{i}^{n}\right) .
\end{aligned}
$$

Thus, we acquire

$$
\begin{aligned}
v_{i}^{n+1}= & v_{i}^{n}+\frac{\beta_{1}}{\operatorname{Re}} t^{\beta_{1}-1} \\
& \cdot\left(\frac{v_{i+1}^{n}-2 v_{i}^{n}+v_{i-1}^{n}}{(\Delta y)^{2}}-\frac{\left.v_{i+2}^{n}-4 v_{i+1}^{n}+6 v_{i}^{n}-4 v_{i-1}^{n}+v_{i-2}^{n}-M_{11} v_{i}^{n}\right)}{(\Delta y)^{4}}\right. \\
& \cdot\left(\frac{3\left(1-\alpha_{1}\right)+3 h \alpha_{1}}{2 M\left(\alpha_{1}\right)}\right)-\frac{\beta_{1}}{\operatorname{Re}} t_{n-1}^{\beta_{1}-1} \\
& \cdot\left(\frac{v_{i+1}^{n-1}-2 v_{i}^{n-1}+v_{i-1}^{n-1}}{(\Delta y)^{2}}-\frac{v_{i+2}^{n-1}-4 v_{i+1}^{n-1}+6 v_{i}^{n-1}-4 v_{i-1}^{n-1}+v_{i-2}^{n-1}}{(\Delta y)^{4}}-M_{11} v_{i}^{n-1}\right) \\
& \cdot\left(\frac{2\left(1-\alpha_{1}\right)+h \alpha_{1}}{2 M\left(\alpha_{1}\right)}\right) .
\end{aligned}
$$

For simplicity, we let

$$
\begin{gathered}
A_{n, \alpha_{1}, \beta_{1}}=\frac{\beta_{1} t_{n}^{\beta_{1}-1} N_{\alpha_{1}}, \quad B_{n, \alpha_{1}, \beta_{1}}=\frac{\beta_{1} t_{n}^{\beta_{1}-1} N_{\alpha_{1}}}{\operatorname{Re}(\Delta y)^{2}},}{\operatorname{Re}(\Delta y)^{4}} \\
C_{n, \alpha_{1}, \beta_{1}}=\frac{\beta_{1} t_{j}^{\beta_{1}-1} M_{11} N_{\alpha_{1}}}{\operatorname{Re}},
\end{gathered}
$$

$$
\begin{gathered}
D_{n, \alpha_{1}, \beta_{1}}=\frac{\beta_{1} t_{n-1}^{\beta_{1}-1} K_{\alpha_{1}}}{\operatorname{Re}(\Delta y)^{2}}, \quad E_{n, \alpha_{1}, \beta_{1}}=\frac{\beta_{1} t_{n-1}^{\beta_{1}-1} K_{\alpha_{1}}}{\operatorname{Re}(\Delta y)^{4}}, \\
F_{n, \alpha_{1}, \beta_{1}}=\frac{\beta_{1} t_{n-1}^{\beta_{1}-1} M_{11} K_{\alpha_{1}}}{\operatorname{Re}},
\end{gathered}
$$

$$
N_{\alpha_{1}}=\frac{2\left(1-\alpha_{1}\right)+3 h \alpha_{1}}{2 M\left(\alpha_{1}\right)}, \quad K_{\alpha_{1}}=\frac{2\left(1-\alpha_{1}\right)+h \alpha_{1}}{2 M\left(\alpha_{1}\right)}
$$

We choose $\varepsilon_{i}^{n}=\delta_{n} \exp \left(i k_{m} y\right) .$. Therefore, we reach

$$
\begin{aligned}
& \delta_{n+1} \exp \left(i k_{m} y\right)=\delta_{n} \exp \left(i k_{m} y\right)-C_{n, n}^{\alpha_{1}, \beta_{1}} \delta_{n} \exp \left(i k_{m} y\right)+A_{n, \alpha_{1}, \beta_{1}}\left[\delta_{n} \exp \left(i k_{m}(y+\Delta y)\right)\right. \\
& \left.-2 \delta_{n} \exp \left(i k_{m} y\right)+\delta_{n} \exp \left(i k_{m}(y-\Delta y)\right)\right]-B_{n, \alpha_{1}, \beta_{1}} \\
& \cdot\left(\begin{array}{c}
\delta_{n} \exp \left(i k_{m}(y+2 \Delta y)\right)-4 \delta_{n} \exp \left(i k_{m}(y+\Delta y)\right)+6 \delta_{n} \exp \left(i k_{m} y\right) \\
-4 \delta_{n} \exp \left(i k_{m}(y-\Delta y)\right)+\delta_{n} \exp \left(i k_{m}(y-2 \Delta y)\right)
\end{array}\right) \\
& -D_{n, \alpha_{1}, \beta_{1}}\left[\delta_{n-1} \exp \left(i k_{m}(y+\Delta y)\right)-2 \delta_{n-1} \exp \left(i k_{m} y\right)+\delta_{n-1} \exp \left(i k_{m}(y-\Delta y)\right)\right] \\
& -E_{n, \alpha_{1}, \beta_{1}}\left(\begin{array}{c}
\delta_{n-1} \exp \left(i k_{m}(y+2 \Delta y)\right)-4 \delta_{n-1} \exp \left(i k_{m}(y+\Delta y)\right)+6 \delta_{n-1} \exp \left(i k_{m} y\right) \\
-4 \delta_{n-1} \exp \left(i k_{m}(y-\Delta y)\right)+\delta_{n-1} \exp \left(i k_{m}(y-2 \Delta y)\right)
\end{array}\right) \\
& +F_{n, n-1}^{\alpha_{1}, \beta_{1}}\left(\delta_{n-1} \exp \left(i k_{m} y\right)\right) \text {. }
\end{aligned}
$$

After simplification, we obtain

$$
\begin{aligned}
\delta_{n+1}= & \delta_{n}-C_{n, \alpha_{1}, \beta_{1}} \delta_{n}+F_{n, \alpha_{1}, \beta_{1}} \delta_{n-1}+A_{n, \alpha_{1}, \beta_{1}}\left[\delta_{n} \exp \left(i k_{m} \Delta y\right)\right. \\
& \left.-2 \delta_{n}+\delta_{n} \exp \left(-i k_{m} \Delta y\right)\right]-B_{n, \alpha_{1}, \beta_{1}} \\
& \cdot\left(\begin{array}{c}
\delta_{n} \exp \left(2 i k_{m} \Delta y\right)-4 \delta_{n} \exp \left(i k_{m} \Delta y\right)+6 \delta_{n} \\
-4 \delta_{n} \exp \left(i k_{m} \Delta y\right)+\delta_{n} \exp \left(-2 i k_{m} \Delta y\right)
\end{array}\right) \\
& -D_{n, \alpha_{1}, \beta_{1}}\left[\delta_{n-1} \exp \left(i k_{m} \Delta y\right)-2 \delta_{n-1}+\delta_{n-1} \exp \left(i k_{m} \Delta y\right)\right] \\
& +E_{n, \alpha_{1}, \beta_{1}}\left(\begin{array}{c}
\delta_{n-1} \exp \left(2 i k_{m} \Delta y\right)-4 \delta_{n-1} \exp \left(i k_{m} \Delta y\right)+6 \delta_{n-1} \\
-4 \delta_{n-1} \exp \left(i k_{m} \Delta y\right)+\delta_{n-1} \exp \left(-2 i k_{m} \Delta y\right)
\end{array}\right) .
\end{aligned}
$$


Thus, we have

$$
\begin{aligned}
\delta_{n+1}= & \delta_{n}\left(1-4 A_{n, \alpha_{1}, \beta_{1}} \sin ^{2}\left(\frac{k_{m} \Delta y}{2}\right)+4 B_{n, j}^{\alpha_{1}, \beta_{1}}\right. \\
& \left.\cdot\left(\sin ^{2}\left(k_{m} \Delta y\right)+4 \sin ^{2}\left(\frac{k_{m} \Delta y}{2}\right)\right)-C_{n, \alpha_{1}, \beta_{1}}\right)+\delta_{n-1} \\
& \cdot\left(4 D_{n, \alpha_{1}, \beta_{1}} \sin ^{2}\left(\frac{k_{m} \Delta y}{2}\right)-4 E_{n, \alpha_{1}, \beta_{1}}\right. \\
& \left.\cdot\left(\sin ^{2}\left(k_{m} \Delta y\right)+4 \sin ^{2}\left(\frac{k_{m} \Delta y}{2}\right)\right)+F_{n, \alpha_{1}, \beta_{1}}\right)
\end{aligned}
$$

For $n=0$, we get

$$
\begin{aligned}
\delta_{1}= & \delta_{0}\left(1-4 A_{0, \alpha_{1}, \beta_{1}} \sin ^{2}\left(\frac{k_{m} \Delta y}{2}\right)+4 B_{0, \alpha_{1}, \beta_{1}}\right. \\
& \left.\cdot\left(\sin ^{2}\left(k_{m} \Delta y\right)+4 \sin ^{2}\left(\frac{k_{m} \Delta y}{2}\right)\right)-C_{0, \alpha_{1}, \beta_{1}}\right) .
\end{aligned}
$$

The $\left|\delta_{1} / \delta_{0}\right|<1$ implies

$$
\left|1-4 A_{0, \alpha_{1}, \beta_{1}} \sin ^{2}\left(\frac{k_{m} \Delta y}{2}\right)+4 B_{0, \alpha_{1}, \beta_{1}}\left(\sin ^{2}\left(k_{m} \Delta y\right)+4 \sin ^{2}\left(\frac{k_{m} \Delta y}{2}\right)\right)-C_{0, \alpha_{1}, \beta_{1}}\right|<1 .
$$

This is true for all $m$.. Thus, we get

$$
\left|1-4 A_{0, \alpha_{1}, \beta_{1}}+20 B_{0, \alpha_{1}, \beta_{1}}-C_{0, \alpha_{1}, \beta_{1}}\right|<1
$$

We assume that $\left|\delta_{n} / \delta_{0}\right|<1$.. Thus, we need to show $\mid$ $\delta_{n+1} / \delta_{0} \mid<1$.

$$
\begin{aligned}
\left|\delta_{n+1}\right| \leq & \left|\delta_{n}\right| \mid 1-4 A_{n, \alpha_{1}, \beta_{1}} \sin ^{2}\left(\frac{k_{m} \Delta y}{2}\right)+4 B_{n, \alpha_{1}, \beta_{1}}\left(\sin ^{2}\left(k_{m} \Delta y\right)+4 \sin ^{2}\left(\frac{k_{m} \Delta y}{2}\right)\right) \\
& -C_{n, \alpha_{1}, \beta_{1}}|+| \delta_{n-1}|| 4 D_{n, \alpha_{1}, \beta_{1}} \sin ^{2}\left(\frac{k_{m} \Delta y}{2}\right)+4 E_{n, \alpha_{1}, \beta_{1}} \\
& \cdot\left(\sin ^{2}\left(k_{m} \Delta y\right)+4 \sin ^{2}\left(\frac{k_{m} \Delta y}{2}\right)\right)+F_{n, \alpha_{1}, \beta_{1}}|<| \delta_{0} \mid\left[\mid 1-4 A_{n, \alpha_{1}, \beta_{1}} \sin ^{2}\right. \\
& \cdot\left(\frac{k_{m} \Delta y}{2}\right)+4 B_{n, \alpha_{1}, \beta_{1}}\left(\sin ^{2}\left(k_{m} \Delta y\right)+4 \sin ^{2}\left(\frac{k_{m} \Delta y}{2}\right)\right) \\
& -C_{n, \alpha_{1}, \beta_{1}}|+| 4 D_{n, \alpha_{1}, \beta_{1}} \sin ^{2}\left(\frac{k_{m} \Delta y}{2}\right)-4 E_{n, \alpha_{1}, \beta_{1}} \\
& \cdot\left(\sin ^{2}\left(k_{m} \Delta y\right)+4 \sin ^{2}\left(\frac{k_{m} \Delta y}{2}\right)\right)+F_{n, \alpha_{1}, \beta_{1}} \mid . "
\end{aligned}
$$

Thus, we obtain

$$
\begin{aligned}
\mid 1 & -4 A_{n, \alpha_{1}, \beta_{1}} \sin ^{2}\left(\frac{k_{m} \Delta y}{2}\right)+4 B_{n, \alpha_{1}, \beta_{1}}\left(\sin ^{2}\left(k_{m} \Delta y\right)+4 \sin ^{2}\left(\frac{k_{m} \Delta y}{2}\right)\right) \\
& -C_{n, \alpha_{1}, \beta_{1}}|+| 4 D_{n, \alpha_{1}, \beta_{1}} \sin ^{2}\left(\frac{k_{m} \Delta y}{2}\right)-4 E_{n, \alpha_{1}, \beta_{1}} \\
& \left(\sin ^{2}\left(k_{m} \Delta y\right)+4 \sin ^{2}\left(\frac{k_{m} \Delta y}{2}\right)\right)+F_{n, \alpha_{1}, \beta_{1}} \mid<1 .
\end{aligned}
$$

This inequality is true for all $m$.. Thus, we get

$$
\begin{gathered}
\left(\left|\left(1-4 A_{n, \alpha_{1}, \beta_{1}}+20 B_{n, \alpha_{1}, \beta_{1}}-C_{n, \alpha_{1}, \beta_{1}}\right)\right|+\mid\right. \\
\left.\quad \cdot\left(4 D_{n, \alpha_{1}, \beta_{1}}-20 E_{n, \alpha_{1}, \beta_{1}}+F_{n, \alpha_{1}, \beta_{1}}\right) \mid\right)<1 .
\end{gathered}
$$

\section{Solution of the Problem with the Generalized Mittag-Leffler Kernel}

We take into consideration the Eq. (10) with fractalfractional differential operator using Definition 3 of Mittag-Leffler kernel as

$$
\begin{gathered}
{ }_{0}^{F F M} D_{t}^{\alpha_{1}, \beta_{1}} v(y, t)=\frac{1}{\operatorname{Re}}\left(\frac{\partial^{2} v(y, t)}{\partial y^{2}}-\frac{\partial^{4} v(y, t)}{\partial y^{4}}-M_{11} v(y, t)\right) \\
\frac{A B\left(\alpha_{1}\right)}{\left(1-\alpha_{1}\right)} \frac{d}{d t} \int_{0}^{t} v(y, \lambda) E_{\alpha_{1}}\left(-\frac{\alpha_{1}}{1-\alpha_{1}}(t-\lambda)^{\alpha_{1}}\right) d \lambda \\
=\frac{\beta_{1}}{\operatorname{Re}} t^{\beta_{1}-1}\left(\frac{\partial^{2} v(y, t)}{\partial y^{2}}-\frac{\partial^{4} v(y, t)}{\partial y^{4}}-M_{11} v(y, t)\right) .
\end{gathered}
$$

For simplicity, we define

$F(y, t, v(y, t))=\frac{\beta_{1}}{\operatorname{Re}} t^{\beta_{1}-1}\left(\frac{\partial^{2} v(y, t)}{\partial y^{2}}-\frac{\partial^{4} v(y, t)}{\partial y^{4}}-M_{11} v(y, t)\right)$.

Then, we get

$v(y, \lambda)=\frac{1-\alpha_{1}}{A B\left(\alpha_{1}\right)} F(y, t, v(y, t))+\frac{\alpha_{1}}{\Gamma\left(\alpha_{1}\right) A B\left(\alpha_{1}\right)} \int_{0}^{t} F(y, \lambda, v(y, \lambda)) d \lambda$.

We discretize above Eq. (53) at $\left(y_{i}, t_{n+1}\right)$ as

$v_{i}^{n+1}=\frac{1-\alpha_{1}}{A B\left(\alpha_{1}\right)} F\left(y_{i}, t_{n+1}, v_{i}^{n}\right)+\frac{\alpha_{1}}{\Gamma\left(\alpha_{1}\right) A B\left(\alpha_{1}\right)} \int_{0}^{t_{n+1}} F\left(y_{i}, \lambda, v\left(y_{i}, \lambda\right)\right)\left(t_{n+1}, \lambda\right)^{\alpha_{1}-1} d \lambda$.

Then, we obtain

$$
\begin{aligned}
v_{i}^{n+1}= & \frac{1-\alpha_{1}}{A B\left(\alpha_{1}\right)} F\left(y_{i}, t_{n+1}, v_{i}^{n}\right)+\frac{\alpha_{1}}{A B\left(\alpha_{1}\right)} \sum_{j=0}^{n} \\
& \cdot\left[\frac{h^{\alpha_{1}} F\left(y_{i}, t_{j}, v_{i}^{j}\right)}{\Gamma\left(\alpha_{1}+2\right)}\left((n+1-j)^{\alpha_{1}}\left(n-j+2+\alpha_{1}\right)-(n-j)^{\alpha_{1}}\left(n-j+2+2 \alpha_{1}\right)\right)\right] \\
& -\frac{\alpha_{1}}{A B\left(\alpha_{1}\right)} \sum_{j=0}^{n}\left[\frac{h^{\alpha_{1}} F\left(y_{i}, t_{j-1}, v_{i}^{j-1}\right)}{\operatorname{Re} \Gamma\left(\alpha_{1}+2\right)}\left((n+1-j)^{\alpha_{1}+1}-(n-j)^{\alpha_{1}}\left(n-j+1+\alpha_{1}\right)\right)\right] .
\end{aligned}
$$

We have

$F\left(y_{i}, t_{j}, v_{i}^{n}\right)=\beta_{1} t_{n}^{\beta_{1}-1}\left(\frac{v_{i+1}^{j}-2 v_{i}^{j}+v_{i-1}^{j}}{(\Delta y)^{2}}-\frac{v_{i+2}^{j}-4 v_{i+1}^{j}+6 v_{i}^{j}-4 v_{i-1}^{j}+v_{i-2}^{j}}{(\Delta y)^{4}}-M_{11} v_{i}^{j}\right)$. 
Then, we will obtain

$$
\begin{aligned}
v_{i}^{n+!}= & \frac{\beta_{1}\left(1-\alpha_{1}\right)}{\operatorname{Re} A B\left(\alpha_{1}\right)} t_{j}^{\beta_{1}-1} \\
& \cdot\left(\frac{v_{i+1}^{j}-2 v_{i}^{j}+v_{i-1}^{j}}{(\Delta y)^{2}}-\frac{v_{i+2}^{j}-4 v_{i+1}^{j}+6 v_{i}^{j}-4 v_{i-1}^{j}+v_{i-2}^{j}}{(\Delta y)^{4}}-M_{11} v_{i}^{j}\right) \\
& +\sum_{j=0}^{n}\left[\frac{h^{\alpha_{1}} \beta_{1} t_{j}^{\beta_{1}-1}}{\Gamma\left(\alpha_{1}+2\right)}\left(\frac{v_{i+1}^{j}-2 v_{i}^{j}+v_{i-1}^{j}}{(\Delta y)^{2}}-\frac{v_{i+2}^{j}-4 v_{i+1}^{j}+6 v_{i}^{j}-4 v_{i-1}^{j}+v_{i-2}^{j}}{(\Delta y)^{4}}-M_{11} j_{i}^{j}\right)\right. \\
& \left.\times\left((n+1-j)^{\alpha_{1}}\left(n-j+2+\alpha_{1}\right)-(n-j)^{\alpha_{1}}\left(n-j+2+2 \alpha_{1}\right)\right)\right] \frac{\alpha_{1}}{\operatorname{Re} A B\left(\alpha_{1}\right)}-\sum_{j=0}^{n} \\
& \cdot\left[\frac{h^{\alpha_{1}} \beta_{1} t_{j-1}^{\beta_{1}-1}}{\Gamma\left(\alpha_{1}+2\right)}\left(\frac{v_{i+1}^{j-1}-2 v_{i}^{j-1}+v_{i-1}^{j-1}}{(\Delta y)^{2}}-\frac{v_{i+2}^{j-1}-4 v_{i+1}^{j-1}+6 v_{i}^{j-1}-4 v_{i-1}^{j-1}+v_{i-2}^{j-1}}{(\Delta y)^{4}}-M_{11} v_{i}^{j-1}\right)\right. \\
& \left.\times\left((n+1-j)^{\alpha_{1}+1}-(n-j)^{\alpha_{1}}\left(n-j+1+\alpha_{1}\right)\right)\right] \frac{\alpha_{1}}{\operatorname{Re} A B\left(\alpha_{1}\right)} .
\end{aligned}
$$

For simplicity, we let

$$
\begin{aligned}
& M_{\alpha_{1}}=\frac{\beta_{1} t_{j}^{\beta_{1}-1}\left(1-\alpha_{1}\right)}{\operatorname{Re}(\Delta y)^{2} A B\left(\alpha_{1}\right)}, \quad K_{\alpha_{1}}=\frac{\beta_{1} t_{j}^{\beta_{1}-1} M_{11}\left(1-\alpha_{1}\right)}{\operatorname{Re} A B\left(\alpha_{1}\right)}, \\
& T_{\alpha_{1}}=\frac{\beta_{1} t_{j}^{\beta_{1}-1}\left(1-\alpha_{1}\right)}{\operatorname{Re}(\Delta y)^{4} A B\left(\alpha_{1}\right)} \\
& A_{n, j}^{\alpha_{1}, \beta_{1}}=\alpha_{1} \frac{h^{\alpha_{1}} \beta_{1} t_{j}^{\beta_{1}-1} K_{n, j}^{\alpha_{1}}}{\Gamma\left(\alpha_{1}+2\right) \operatorname{Re}(\Delta y)^{2} A B\left(\alpha_{1}\right)}, \\
& B_{n, j}^{\alpha_{1}, \beta_{1}}=\alpha_{1} \frac{h^{\alpha_{1}} \beta_{1} t_{j}^{\beta_{1}-1} K_{n, j}^{\alpha_{1}}}{\Gamma\left(\alpha_{1}+2\right) \operatorname{Re}(\Delta y)^{4} A B\left(\alpha_{1}\right)} \text {, } \\
& C_{n, j}^{\alpha_{1}, \beta_{1}}=\alpha_{1} \frac{h^{\alpha_{1}} \beta_{1} t_{j}^{\beta_{1}-1} M_{11} K_{n, j}^{\alpha_{1}}}{\Gamma\left(\alpha_{1}+2\right) \operatorname{Re} A B\left(\alpha_{1}\right)}, \\
& A_{n, j-1}^{\alpha_{1}, \beta_{1}}=\alpha_{1} \frac{h^{\alpha_{1}} \beta_{1} t_{j-1}^{\beta_{1}-1} \gamma_{n, j}^{\alpha_{1}}}{\Gamma\left(\alpha_{1}+2\right) \operatorname{Re}(\Delta y)^{2} A B\left(\alpha_{1}\right)}, \\
& B_{n, j-1}^{\alpha_{1}, \beta_{1}}=\alpha_{1} \frac{h^{\alpha_{1}} \beta_{1} t_{j-1}^{\beta_{1}-1} \gamma_{n, j}^{\alpha_{1}}}{\Gamma\left(\alpha_{1}+2\right) \operatorname{Re}(\Delta y)^{4} A B\left(\alpha_{1}\right)}, \\
& C_{n, j-1}^{\alpha_{1}, \beta_{1}}=\alpha_{1} \frac{h^{\alpha_{1}} \beta_{1} t_{j-1}^{\beta_{1}-1} M_{11} K_{n, j}^{\alpha_{1}}}{\Gamma\left(\alpha_{1}+2\right) \operatorname{Re} A B\left(\alpha_{1}\right)} \text {, } \\
& K_{n, j}^{\alpha_{1}}=\left((n+1-j)^{\alpha_{1}}\left(n-j+2+\alpha_{1}\right)-(n-j)^{\alpha_{1}}\left(n-j+2+2 \alpha_{1}\right)\right), \\
& \gamma_{n, j}^{\alpha_{1}}=\left((n+1-j)^{\alpha_{1}+1}-(n-j)^{\alpha_{1}}\left(n-j+1+\alpha_{1}\right)\right) .
\end{aligned}
$$

Then, we get

$$
\begin{aligned}
v_{i}^{n+1}= & M_{\alpha_{1}}\left(v_{i+1}^{n}-2 v_{i}^{n}+v_{i-1}^{n}\right)-K_{\alpha_{1}} v_{i}^{n}-T_{\alpha_{1}} \\
& \cdot\left(v_{i+2}^{n}-4 v_{i+1}^{n}+6 v_{i}^{n}-4 v_{i-1}^{n}+v_{i-2}^{n}\right) \\
& +\sum_{j=0}^{n}\left[A_{n, j}^{\alpha_{1}, \beta_{1}}\left(v_{i+1}^{j}-2 v_{i}^{j}+v_{i-1}^{j}\right)-B_{n, j}^{\alpha_{1}, \beta_{1}}\right. \\
& \left.\cdot\left(v_{i+2}^{j}-4 v_{i+1}^{j}+6 v_{i}^{j}-4 v_{i-1}^{j}+v_{i-2}^{j}\right)-C_{n, j}^{\alpha_{1}, \beta_{1}} v_{i}^{j}\right] \\
& -\sum_{j=0}^{n}\left[A_{n, j-1}^{\alpha_{1}, \beta_{1}}\left(v_{i+1}^{j-1}-2 v_{i}^{j-1}+v_{i-1}^{j-1}\right)-B_{n, j-1}^{\alpha_{1}, \beta_{1}}\right. \\
& \left.\cdot\left(v_{i+2}^{j-1}-4 v_{i+1}^{j-1}+6 v_{i}^{j-1}-4 v_{i-1}^{j-1}+v_{i-2}^{j-1}\right)-C_{n, j-1}^{\alpha_{1}, \beta_{1}}\left(v_{i}^{j-1}\right)\right] .
\end{aligned}
$$

We choose $\varepsilon_{i}^{n}=\delta_{n} \exp \left(i k_{m} y\right)$.. Then, we acquire

$$
\begin{aligned}
& \delta_{n+1} \exp \left(i k_{m} y\right)=M_{\alpha_{1}}\left[\delta_{n} \exp \left(i k_{m}(y+\Delta y)\right)-2 \delta_{n} \exp \left(i k_{m} y\right)+\delta_{n} \exp \left(i k_{m}(y-\Delta y)\right)\right] \\
& -K_{\alpha_{1}} \delta_{n} \exp \left(i k_{m} y\right)-T_{\alpha_{1}}\left(\delta_{n} \exp \left(i k_{m}(y+2 \Delta y)\right)-4 \delta_{n} \exp \left(i k_{m}(y+\Delta y)\right)\right. \\
& \left.+6 \delta_{n} \exp \left(i k_{m} y\right)-4 \delta_{n} \exp \left(i k_{m}(y-\Delta y)\right)+\delta_{n} \exp \left(i k_{m}(y-2 \Delta y)\right)\right) \\
& +A_{n, n}^{\alpha_{1}, \beta_{1}}\left[\delta_{n} \exp \left(i k_{m}(y+\Delta y)\right)-2 \delta_{n} \exp \left(i k_{m} y\right)+\delta_{n} \exp \left(i k_{m}(y-\Delta y)\right)\right] \\
& -B_{n, n}^{\alpha_{1}, \beta_{1}}\left(\begin{array}{c}
\delta_{n} \exp \left(i k_{m}(y+2 \Delta y)\right)-4 \delta_{n} \exp \left(i k_{m}(y+\Delta y)\right)+6 \delta_{n} \exp \left(i k_{m} y\right) \\
-4 \delta_{n} \exp \left(i k_{m}(y-\Delta y)\right)+\delta_{n} \exp \left(i k_{m}(y-2 \Delta y)\right)
\end{array}\right) \\
& -C_{n, n}^{\alpha_{1}, \beta_{1}} \delta_{n} \exp \left(i k_{m} y\right)-A_{n, n-1}^{\alpha_{1}, \beta_{1}}\left[\delta_{n-1} \exp \left(i k_{m}(y+\Delta y)\right)-2 \delta_{n-1} \exp \left(i k_{m} y\right)\right. \\
& \left.+\delta_{n-1} \exp \left(i k_{m}(y-\Delta y)\right)\right]+B_{n, n-1}^{\alpha_{1}, \beta_{1}} \\
& \cdot\left(\begin{array}{c}
\delta_{n-1} \exp \left(i k_{m}(y+2 \Delta y)\right)-4 \delta_{n-1} \exp \left(i k_{m}(y+\Delta y)\right)+6 \delta_{n-1} \exp \left(i k_{m} y\right) \\
-4 \delta_{n-1} \exp \left(i k_{m}(y-\Delta y)\right)+\delta_{n-1} \exp \left(i k_{m}(y-2 \Delta y)\right)
\end{array}\right) \\
& +C_{n, n-1}^{\alpha_{1}, \beta_{1}}\left(\delta_{n-1} \exp \left(i k_{m} y\right)\right)+\sum_{j=0}^{n-1}
\end{aligned}
$$

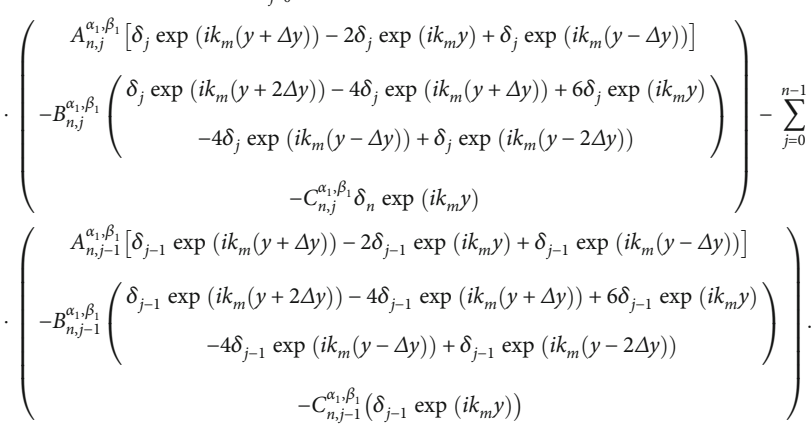

Then, we get

$$
\begin{aligned}
& \delta_{n+1}=M_{\alpha_{1}}\left[\delta_{n} \exp \left(i k_{m} \Delta y\right)-2 \delta_{n}+\delta_{n} \exp \left(-i k_{m} \Delta y\right)\right]-K_{\alpha_{1}} \delta_{n}-T_{\alpha_{1}}\left(\delta_{n} \exp \left(2 i k_{m} \Delta y\right)\right. \\
& \left.-4 \delta_{n} \exp \left(i k_{m} \Delta y\right)+6 \delta_{n}-4 \delta_{n} \exp \left(-i k_{m} \Delta y\right)+\delta_{n} \exp \left(-2 i k_{m} \Delta y\right)\right) \\
& +A_{n, n}^{\alpha_{1}, \beta_{1}}\left[\delta_{n} \exp \left(i k_{m} \Delta y\right)-2 \delta_{n}+\delta_{n} \exp \left(-i k_{m} \Delta y\right)\right] \\
& -B_{n, n}^{\alpha_{1}, \beta_{1}}\left(\begin{array}{c}
\delta_{n} \exp \left(2 i k_{m} \Delta y\right)-4 \delta_{n} \exp \left(i k_{m} \Delta y\right)+6 \delta_{n} \\
-4 \delta_{n} \exp \left(i k_{m} \Delta y\right)+\delta_{n} \exp \left(-2 i k_{m} \Delta y\right)
\end{array}\right)-C_{n, n}^{\alpha_{1}, \beta_{1}} \delta_{n} \\
& -A_{n, n-1}^{\alpha_{1}, \beta_{1}}\left[\delta_{n-1} \exp \left(i k_{m} \Delta y\right)-2 \delta_{n-1}+\delta_{n-1} \exp \left(i k_{m} \Delta y\right)\right] \\
& +B_{n, n-1}^{\alpha_{1}, \beta_{1}}\left(\begin{array}{c}
\delta_{n-1} \exp \left(2 i k_{m} \Delta y\right)-4 \delta_{n-1} \exp \left(i k_{m} \Delta y\right)+6 \delta_{n-1} \\
-4 \delta_{n-1} \exp \left(i k_{m} \Delta y\right)+\delta_{n-1} \exp \left(-2 i k_{m} \Delta y\right)
\end{array}\right)
\end{aligned}
$$

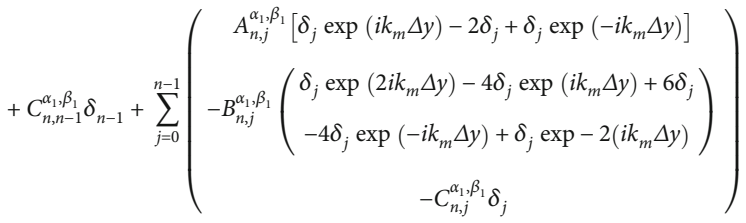

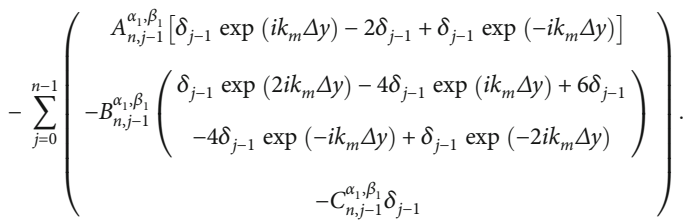

We prove by induction. For $n=0$, we have 


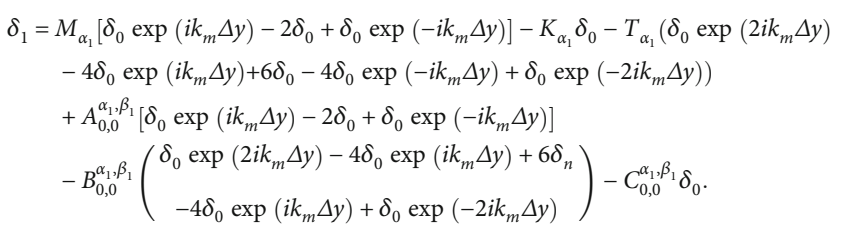

Then, we get

$$
\begin{aligned}
\delta_{1}= & \delta_{0} M_{\alpha_{1}}\left[\exp \left(i k_{m} \Delta y\right)-2+\exp \left(-i k_{m} \Delta y\right)\right]-K_{\alpha_{1}} \delta_{0}-\delta_{0} T_{\alpha_{1}}\left(\exp \left(2 i k_{m} \Delta y\right)\right. \\
& \left.-4 \exp \left(i k_{m} \Delta y\right)+6-4 \exp \left(-i k_{m} \Delta y\right)+\exp \left(-2 i k_{m} \Delta y\right)\right) \\
& +\delta_{0} A_{0,0}^{\alpha_{1}, \beta_{1}}\left[\exp \left(i k_{m} \Delta y\right)-2+\exp \left(-i k_{m} \Delta y\right)\right] \\
& -\delta_{0} B_{0,0}^{\alpha_{1}, \beta_{1}}\left(\begin{array}{c}
\exp \left(2 i k_{m} \Delta y\right)-4 \exp \left(i k_{m} \Delta y\right)+6 \\
-4 \exp \left(i k_{m} \Delta y\right)+\exp \left(-2 i k_{m} \Delta y\right)
\end{array}\right)-C_{0,0}^{\alpha_{1}, \beta_{1}} \delta_{0} .
\end{aligned}
$$

Thus, we reach

$$
\begin{aligned}
\delta_{1}= & \delta_{0} M_{\alpha_{1}}\left[-4 \sin ^{2}\left(\frac{k_{m} \Delta y}{2}\right)\right]-K_{\alpha_{1}} \delta_{0}+4 \delta_{0} T_{\alpha_{1}}\left(\sin ^{2}\left(\frac{k_{m} \Delta y}{2}\right)+4 \sin ^{2}\left(\frac{k_{m} \Delta y}{2}\right)\right) \\
& +\delta_{0}\left(-4 \sin ^{2}\left(\frac{k_{m} \Delta y}{2}\right) A_{0,0}^{\alpha_{1}, \beta_{1}}+4 B_{0,0}^{\alpha_{1}, \beta_{1}}\left(\sin ^{2}\left(k_{m} \Delta y\right)+4 \sin ^{2}\left(\frac{k_{m} \Delta y}{2}\right)\right)-C_{0,0}^{\alpha_{1}, \beta_{1}}\right) .
\end{aligned}
$$

After simplification, we obtain

$\delta_{1}=\delta_{0}\left(\begin{array}{c}-4 \sin ^{2}\left(\frac{k_{m} \Delta y}{2}\right)\left(M_{\alpha_{1}}+A_{0,0}^{\alpha_{1}, \beta_{1}}\right)-K_{\alpha_{1}}-C_{0,0}^{\alpha_{1}, \beta_{1}} \\ +4\left(T_{\alpha_{1}}+B_{0,0}^{\alpha_{1}, \beta_{1}}\right)\left(\sin ^{2}\left(\frac{k_{m} \Delta y}{2}\right)+4 \sin ^{2}\left(\frac{k_{m} \Delta y}{2}\right)\right)\end{array}\right)$.

We should show $\left|\delta_{1} / \delta_{0}\right|<1$.. Therefore, we have

$$
\left|\begin{array}{c}
-4 \sin ^{2}\left(\frac{k_{m} \Delta y}{2}\right)\left(M_{\alpha_{1}}+A_{0,0}^{\alpha_{1}, \beta_{1}}\right)-K_{\alpha_{1}}-C_{0,0}^{\alpha_{1}, \beta_{1}} \\
+4\left(T_{\alpha_{1}}+B_{0,0}^{\alpha_{1}, \beta_{1}}\right)\left(\sin ^{2}\left(\frac{k_{m} \Delta y}{2}\right)+4 \sin ^{2}\left(\frac{k_{m} \Delta y}{2}\right)\right)
\end{array}\right|
$$

Since we have for all $m$, we obtain

$$
\left|-4\left(M_{\alpha_{1}}+A_{0,0}^{\alpha_{1}, \beta_{1}}\right)-K_{\alpha_{1}}-C_{0,0}^{\alpha_{1}, \beta_{1}}+20\left(T_{\alpha_{1}}+B_{0,0}^{\alpha_{1}, \beta_{1}}\right)\right|<1 .
$$

When $n>1$, we have

$$
\begin{array}{r}
\delta_{n+1}=M_{\alpha_{1}}\left[\delta_{n} \exp \left(i k_{m} \Delta y\right)-2 \delta_{n}+\delta_{n} \exp \left(-i k_{m} \Delta y\right)\right]-K_{\alpha_{1}} \delta_{n}-T_{\alpha_{1}}\left(\delta_{n} \exp \left(2 i k_{m} \Delta y\right)\right. \\
\left.-4 \delta_{n} \exp \left(i k_{m} \Delta y\right)+6 \delta_{n}-4 \delta_{n} \exp \left(-i k_{m} \Delta y\right)+\delta_{n} \exp \left(-2 i k_{m} \Delta y\right)\right) \\
+\sum_{j=0}^{n}\left(\begin{array}{c}
A_{n, j}^{\alpha_{1}, \beta_{1}}\left[\delta_{j} \exp \left(i k_{m} \Delta y\right)-2 \delta_{j}+\delta_{j} \exp \left(-i k_{m} \Delta y\right)\right] \\
-B_{n, j}^{\alpha_{1}, \beta_{1}}\left(\begin{array}{c}
\delta_{j} \exp \left(2 i k_{m} \Delta y\right)-4 \delta_{j} \exp \left(i k_{m} \Delta y\right)+6 \delta_{j} \\
-4 \delta_{j} \exp \left(-i k_{m} \Delta y\right)+\delta_{j} \exp -2\left(i k_{m} \Delta y\right)
\end{array}\right) \\
-C_{n, j}^{\alpha_{1}, \beta_{1}} \delta_{n}
\end{array}\right) \\
-\sum_{j=0}^{n}\left(\begin{array}{c}
A_{n, j-1}^{\alpha_{1}, \beta_{1}}\left[\delta_{j-1} \exp \left(i k_{m} \Delta y\right)-2 \delta_{j-1}+\delta_{j-1} \exp \left(-i k_{m} \Delta y\right)\right] \\
-B_{n, j-1}^{\alpha_{1}, \beta_{1}}\left(\begin{array}{c}
\delta_{j-1} \exp \left(2 i k_{m} \Delta y\right)-4 \delta_{j-1} \exp \left(i k_{m} \Delta y\right)+6 \delta_{j-1} \\
-4 \delta_{j-1} \exp \left(-i k_{m} \Delta y\right)+\delta_{j-1} \exp \left(-2 i k_{m} \Delta y\right)
\end{array}\right) \\
-C_{n, j-1}^{\alpha_{1}, \beta_{1}} \delta_{j-1}
\end{array}\right) .
\end{array}
$$

We suppose that for all $n \geq 1,\left|\delta_{n} / \delta_{0}\right|<1$.. We want to prove that $\left|\delta_{n+1} / \delta_{0}\right|<1$.. However,

$$
\begin{aligned}
& \left|\delta_{n+1}\right| \leq\left|\delta_{n}\right|\left|-4 \sin ^{2}\left(\frac{k_{m} \Delta y}{2}\right) M_{\alpha_{1}}+K_{\alpha_{1}}\right|+\sum_{j=0}^{n}\left|\delta_{j}\right| \mid \\
& -4 \sin ^{2}\left(\frac{k_{m} \Delta y}{2}\right) A_{n, j}^{\alpha_{1}, \beta_{1}}+4 B_{n, j}^{\alpha_{1}, \beta_{1}}\left(\sin ^{2}\left(k_{m} \Delta y\right)+4 \sin ^{2}\left(\frac{k_{m} \Delta y}{2}\right)\right) \\
& -C_{n, j}^{\alpha_{1}, \beta_{1}}\left|+\sum_{j=0}^{n-1}\right| \delta_{j-1}||-4 \sin ^{2}\left(\frac{k_{m} \Delta y}{2}\right) A_{n, j-1}^{\alpha_{1}, \beta_{1}}+4 B_{n, j-1}^{\alpha_{1}, \beta_{1}} \\
& \cdot\left(\sin ^{2}\left(k_{m} \Delta y\right)+4 \sin ^{2}\left(\frac{k_{m} \Delta y}{2}\right)\right)-C_{n, j-1}^{\alpha_{1}, \beta_{1}} \mid . "
\end{aligned}
$$

By induction hypothesis for all $n \geq 1,\left|\delta_{n}\right|<\left|\delta_{0}\right|$, we have

$$
\begin{aligned}
& \left|\delta_{n+1}\right| \leq\left|\delta_{0}\right|\left|-4 \sin ^{2}\left(\frac{k_{m} \Delta y}{2}\right) M_{\alpha_{1}}+K_{\alpha_{1}}\right|+\sum_{j=0}^{n}\left|\delta_{0}\right| \mid\left(-4 \sin ^{2}\left(\frac{k_{m} \Delta y}{2}\right) A_{n, j}^{\alpha_{1}, \beta_{1}}\right. \\
& \left.\quad+4 B_{n, j}^{\alpha_{1}, \beta_{1}}\left(\sin ^{2}\left(k_{m} \Delta y\right)+4 \sin ^{2}\left(\frac{k_{m} \Delta y}{2}\right)\right)-C_{n, j}^{\alpha_{1}, \beta_{1}}\right) \mid \\
& \quad+\sum_{j=0}^{n}\left|\delta_{0}\right| \mid\left(-4 \sin ^{2}\left(\frac{k_{m} \Delta y}{2}\right) A_{n, j-1}^{\alpha_{1}, \beta_{1}}+4 B_{n, j-1}^{\alpha_{1}, \beta_{1}}\left(\sin ^{2}\left(k_{m} \Delta y\right)+4 \sin ^{2}\left(\frac{k_{m} \Delta y}{2}\right)\right)\right. \\
& \left.\quad-C_{n, j-1}^{\alpha_{1}, \beta_{1}}\right) \mid .
\end{aligned}
$$

This inequality is true for all $m$. Thus, we reach

$$
\begin{aligned}
& \left|\delta_{n+1}\right| \leq\left|\delta_{0}\right|\left|-4 M_{\alpha_{1}}+K_{\alpha_{1}}\right|+\sum_{j=0}^{n}\left|\delta_{0}\right|\left|\left(-4 A_{n, j}^{\alpha_{1}, \beta_{1}}+20 B_{n, j}^{\alpha_{1}, \beta_{1}}-C_{n, j}^{\alpha_{1}, \beta_{1}}\right)\right| \\
& \quad+\sum_{j=0}^{n-1}\left|\delta_{0}\right|\left|\left(-4 A_{n, j-1}^{\alpha_{1}, \beta_{1}}+20 B_{n, j-1}^{\alpha_{1}, \beta_{1}}-C_{n, j-1}^{\alpha_{1}, \beta_{1}}\right)\right| .
\end{aligned}
$$


We need to show that $\left|\delta_{n+1} / \delta_{0}\right|<1$. Thus, we get

$$
\begin{gathered}
\left|-4 M_{\alpha_{1}}+K_{\alpha_{1}}\right|+\sum_{j=0}^{n}\left|\left(-4 A_{n, j}^{\alpha_{1}, \beta_{1}}+20 B_{n, j}^{\alpha_{1}, \beta_{1}}-C_{n, j}^{\alpha_{1}, \beta_{1}}\right)\right| \\
+\sum_{j=0}^{n-1}\left|\left(-4 A_{n, j-1}^{\alpha_{1}, \beta_{1}}+20 B_{n, j-1}^{\alpha_{1}, \beta_{1}}-C_{n, j-1}^{\alpha_{1}, \beta_{1}}\right)\right|<1 .
\end{gathered}
$$

\section{Error Analysis}

In this section, we will consider the error analysis.

$$
\begin{gathered}
{ }_{0}^{F F P} D_{t}^{\alpha_{1}, \beta_{1}} v(y, t)=\frac{1}{\operatorname{Re}}\left(\frac{\partial^{2} v(y, t)}{\partial y^{2}}-\frac{\partial^{4} v(y, t)}{\partial y^{4}}-M_{11} v(y, t)\right), \\
\frac{1}{\Gamma\left(1-\alpha_{1}\right)} \frac{d}{d t} \int_{0}^{t} v(y, t)(t-\lambda)^{-\alpha_{1}} d \lambda \\
=\frac{\beta_{1}}{\operatorname{Re}} t^{\beta_{1}-1}\left(\frac{\partial^{2} v(y, t)}{\partial y^{2}}-\frac{\partial^{4} v(y, t)}{\partial y^{4}}-M_{11} v(y, t)\right)
\end{gathered}
$$

Then, we get

$v(y, t)=\frac{\beta_{1}}{\operatorname{Re} \Gamma\left(\alpha_{1}\right)} \int_{0}^{t} \lambda^{\beta_{1}-1}\left(\frac{\partial^{2} v(y, \lambda)}{\partial y^{2}}-\frac{\partial^{4} v(y, \lambda)}{\partial y^{4}}-M_{11} v(y, \lambda)\right)(t-\lambda)^{\alpha_{1}-1} d \lambda$.

For simplicity, we take

$$
F(y, \lambda)=\beta_{1} \lambda^{\beta_{1}-1}\left(\frac{\partial^{2} v(y, \lambda)}{\partial y^{2}}-\frac{\partial^{4} v(y, \lambda)}{\partial y^{4}}-M_{11} v(y, \lambda)\right) .
$$

Then, we have

$$
v(y, t)=\frac{1}{\operatorname{Re} \Gamma\left(\alpha_{1}\right)} \int_{0}^{t} F(y, \lambda)(t-\lambda)^{\alpha_{1}-1} d \lambda
$$

At $\left(y_{i}, t=t_{n+1}\right)$, we get

$$
\begin{aligned}
v_{i}^{n+1}= & \frac{1}{\Gamma\left(\alpha_{1}\right)} \int_{0}^{t_{n+1}} F\left(y_{i}, \lambda\right)\left(t_{n+1}-\lambda\right)^{\alpha_{1}-1} d \lambda \\
= & \frac{1}{\Gamma\left(\alpha_{1}\right)} \sum_{j=0}^{n} \int_{t_{j}}^{t_{j+1}}\left(P_{j}(\lambda)+\left.\frac{\left(\lambda-t_{j}\right)\left(\lambda-t_{j-1}\right)}{2 !} \frac{\partial^{2}}{\partial \lambda^{2}} F\left(y_{i}, \lambda\right)\right|_{\lambda=\varepsilon_{\lambda}}\right)\left(t_{n+1}-\lambda\right)^{\alpha_{1}-1} d \lambda \\
= & \frac{1}{\Gamma\left(\alpha_{1}\right)} \sum_{j=0}^{n} \int_{t_{j}}^{t_{j+1}} P_{j}(\lambda)\left(t_{n+1}-\lambda\right)^{\alpha_{1}-1} d \lambda+\frac{1}{\Gamma\left(\alpha_{1}\right)} \sum_{j=0}^{n} \int_{t_{j}}^{t_{j+1}} \\
& \cdot\left(\left.\frac{\left(\lambda-t_{j}\right)\left(\lambda-t_{j-1}\right)}{2 !} \frac{\partial^{2}}{\partial \lambda^{2}} F\left(y_{i}, \lambda\right)\right|_{\lambda=\varepsilon_{\lambda}}\right)\left(t_{n+1}-\lambda\right)^{\alpha_{1}-1} d \lambda \\
= & \frac{1}{\Gamma\left(\alpha_{1}\right)} \sum_{j=0}^{n} \int_{t_{j}}^{t_{j+1}} P_{j}(\lambda)\left(t_{n+1}-\lambda\right)^{\alpha_{1}-1} d \lambda+R_{n, j}^{\alpha_{1}},
\end{aligned}
$$

$$
\begin{aligned}
R_{n, j}^{\alpha_{1}}= & \frac{1}{\Gamma\left(\alpha_{1}\right)} \sum_{j=0}^{n} \int_{t_{j}}^{t_{j+1}}\left(\frac{\partial^{2}}{\partial \lambda^{2}}\left[\beta_{1} \lambda^{\beta_{1}-1}\left(\frac{\partial^{2} v(y, \lambda)}{\partial y^{2}}-\frac{\partial^{4} v(y, \lambda)}{\partial y^{4}}-M_{11} v(y, \lambda)\right)\right]\right) \\
& \cdot\left(t_{n+1}-\lambda\right)^{\alpha_{1}-1} d \lambda \leq \frac{1}{\Gamma\left(\alpha_{1}\right)} \sum_{j=0}^{n} \int_{t_{j}}^{t_{j+1}} \\
& \cdot\left(\begin{array}{l}
\frac{\left|\lambda-t_{j}\right|\left|\lambda-t_{j-1}\right|}{2 !} \\
2 !
\end{array} \mid\left(t_{n+1}-\lambda\right)^{\alpha_{1}-1} d \lambda .\right.
\end{aligned}
$$

Then, we have

$$
\begin{aligned}
& \frac{\partial^{2}}{\partial \lambda^{2}}\left[\beta_{1} \lambda^{\beta_{1}-1}\left(\frac{\partial^{2} v\left(y_{i}, \lambda\right)}{\partial y^{2}}-\frac{\partial^{4} v\left(y_{i}, \lambda\right)}{\partial y^{4}}-M_{11} v\left(y_{i}, \lambda\right)\right)\right] \\
& \quad=\beta_{1}\left[\left(\beta_{1}-1\right)\left(\beta_{1}-2\right) \lambda^{\beta_{1}-3}\left(\frac{\partial^{2} v\left(y_{i}, \lambda\right)}{\partial y^{2}}-\frac{\partial^{4} v\left(y_{i}, \lambda\right)}{\partial y^{4}}-M_{11} v\left(y_{i}, \lambda\right)\right)\right. \\
& \quad+2\left(\beta_{1}-1\right) \lambda^{\beta_{1}-2} \times\left(\frac{\partial^{3} v\left(y_{i}, \lambda\right)}{\partial \lambda \partial y^{2}}-\frac{\partial^{5} v\left(y_{i}, \lambda\right)}{\partial \lambda \partial y^{4}}-M_{11} \frac{\partial v\left(y_{i}, \lambda\right)}{\partial \lambda}\right) \\
& \left.\quad+\lambda^{\beta_{1}-1}\left(\frac{\partial^{4} v\left(y_{i}, \lambda\right)}{\partial \lambda^{2} \partial y^{2}}-\frac{\partial^{6} v\left(y_{i}, \lambda\right)}{\partial \lambda^{2} \partial y^{4}}-M_{11} \frac{\partial^{2} v\left(y_{i}, \lambda\right)}{\partial \lambda^{2}}\right)\right]
\end{aligned}
$$

$$
\begin{aligned}
& \left|R_{n, j}^{\alpha_{1}}\right|<\frac{1}{\Gamma\left(\alpha_{1}\right)} \sum_{j=0}^{n} \int_{t_{j}}^{t_{j+1}} \sup _{\tau \in\left[\tau_{\left.j, \tau_{j+1}\right]}\left|\lambda-t_{j}\right| \sup _{\tau \in\left[\tau_{j-1}, \tau_{j+1}\right]}\left|\lambda-t_{j-1}\right|\right.} \\
& \times \sup _{\tau \in\left[\tau_{j}, \tau_{j+1}\right]}\left|\frac{\partial^{2}}{\partial \lambda^{2}}\left[\beta_{1} \lambda^{\beta_{1}-1}\left(\frac{\partial^{2} v\left(y_{i}, \lambda\right)}{\partial y^{2}}-\frac{\partial^{4} v\left(y_{i}, \lambda\right)}{\partial y^{4}}-M_{11} v\left(y_{i}, \lambda\right)\right)\right]\right| \\
& \cdot\left(t_{n+1}-\lambda\right)^{\alpha_{1}-1} d \lambda<\frac{C_{1} C_{2}}{\Gamma\left(\alpha_{1}\right)} \sum_{j=0}^{n} \int_{t_{j}}^{t_{j+1}} \sup _{\tau \in\left[\tau_{j} \tau_{j+1}\right]} \mid \frac{\partial^{2}}{\partial \lambda^{2}} \\
& \quad \cdot\left[\beta_{1} \lambda^{\beta_{1}-1}\left(\frac{\partial^{2} v\left(y_{i}, \lambda\right)}{\partial y^{2}}-\frac{\partial^{4} v\left(y_{i}, \lambda\right)}{\partial y^{4}}-M_{11} v\left(y_{i}, \lambda\right)\right)\right] \mid\left(t_{n+1}-\lambda\right)^{\alpha_{1}-1} d \lambda .
\end{aligned}
$$

We have

$$
\begin{gathered}
\sup _{\tau \in\left[\tau_{\left.j, \tau_{j+1}\right]}\right]}\left|\frac{\partial^{2}}{\partial \lambda^{2}}\left[\beta_{1} \lambda^{\beta_{1}-1}\left(\frac{\partial^{2} v\left(y_{i}, \lambda\right)}{\partial y^{2}}-\frac{\partial^{4} v\left(y_{i}, \lambda\right)}{\partial y^{4}}-M_{11} v\left(y_{i}, \lambda\right)\right)\right]\right| \\
<\beta_{1}\left[\left|\left(\beta_{1}-1\right)\left(\beta_{1}-2\right)\right| \lambda^{\beta_{1}-3}\left(\left\|\frac{\partial^{2} v}{\partial y^{2}}\right\|_{\infty}+\left\|\frac{\partial^{4} v}{\partial y^{4}}\right\|_{\infty}+\left|M_{11}\right|\|v\|_{\infty}\right)\right. \\
+2\left|\left(\beta_{1}-1\right)\right| \lambda^{\beta_{1}-2} \times\left(\left\|\frac{\partial^{3} v}{\partial \lambda \partial y^{2}}\right\|_{\infty}+\left\|\frac{\partial^{5} v}{\partial \lambda \partial y^{4}}\right\|_{\infty}-M_{11}\left\|\frac{\partial v}{\partial \lambda}\right\|_{\infty}\right) \\
\left.+\lambda^{\beta_{1}-1}\left(\left\|\frac{\partial^{4} v}{\partial \lambda^{2} \partial y^{2}}\right\|_{\infty}+\left\|\frac{\partial^{6} v}{\partial \lambda^{2} \partial y^{4}}\right\|_{\infty}-M_{11}\left\|\frac{\partial^{2} v}{\partial \lambda^{2}}\right\|_{\infty}\right)\right] .
\end{gathered}
$$


Therefore, we acquire

$$
\begin{aligned}
& \left|R_{n, j}^{\alpha_{1}}\right|<\frac{C_{1} C_{2}}{\Gamma\left(\alpha_{1}\right)} \beta_{1}\left[\left|\left(\beta_{1}-1\right)\left(\beta_{1}-2\right)\right| A_{1}\left(\left\|\frac{\partial^{2} v}{\partial y^{2}}\right\|_{\infty}+\left\|\frac{\partial^{4} v}{\partial y^{4}}\right\|_{\infty}+\left|M_{11}\right|\|v\|_{\infty}\right)\right. \\
& +2\left|\left(\beta_{1}-1\right)\right| A_{2}\left(\left\|\frac{\partial^{3} v}{\partial \lambda \partial y^{2}}\right\|_{\infty}+\left\|\frac{\partial^{5} v}{\partial \lambda \partial y^{4}}\right\|_{\infty}+M_{11}\left\|\frac{\partial v}{\partial \lambda}\right\|_{\infty}\right) \\
& \left.+A_{3}\left(\left\|\frac{\partial^{4} v}{\partial \lambda^{2} \partial y^{2}}\right\|_{\infty}+\left\|\frac{\partial^{6} v}{\partial \lambda^{2} \partial y^{4}}\right\|_{\infty}+M_{11}\left\|\frac{\partial^{2} v}{\partial \lambda^{2}}\right\|_{\infty}\right)\right] \sum_{j=0}^{n} \int_{t_{j}}^{t_{j+1}}\left(t_{n+1}-\lambda\right)^{\alpha_{1}-1} d \lambda,
\end{aligned}
$$

where

$$
\frac{1}{\Gamma\left(\alpha_{1}\right)} \sum_{j=0}^{n} \int_{t_{j}}^{t_{j+1}}\left(t_{n+1}-\lambda\right)^{\alpha_{1}-1} d \lambda=\frac{(\Delta t)^{\alpha_{1}}(n+1)^{\alpha_{1}}}{\Gamma\left(1+\alpha_{1}\right)} .
$$

Therefore, we obtain

$$
\begin{gathered}
\left|R_{n, j}^{\alpha_{1}}\right|<\frac{C_{1} C_{2}}{\Gamma\left(\alpha_{1}\right)} \beta_{1}\left[\left|\left(\beta_{1}-1\right)\left(\beta_{1}-2\right)\right| A_{1}\left(\left\|\frac{\partial^{2} v}{\partial y^{2}}\right\|_{\infty}+\left\|\frac{\partial^{4} v}{\partial y^{4}}\right\|_{\infty}+\left|M_{11}\right|\|v\|_{\infty}\right)\right. \\
+2\left|\left(\beta_{1}-1\right)\right| A_{2}\left(\left\|\frac{\partial^{3} v}{\partial \lambda \partial y^{2}}\right\|_{\infty}+\left\|\frac{\partial^{5} v}{\partial \lambda \partial y^{4}}\right\|_{\infty}+M_{11}\left\|\frac{\partial v}{\partial \lambda}\right\|_{\infty}\right) \\
\left.+A_{3}\left(\left\|\frac{\partial^{4} v}{\partial \lambda^{2} \partial y^{2}}\right\|_{\infty}+\left\|\frac{\partial^{6} v}{\partial \lambda^{2} \partial y^{4}}\right\|_{\infty}+M_{11}\left\|\frac{\partial^{2} v}{\partial \lambda^{2}}\right\|_{\infty}\right)\right] \sum_{j=0}^{n} \frac{(\Delta t)^{\alpha_{1}}(n+1)^{\alpha_{1}}}{\Gamma\left(1+\alpha_{1}\right)} .
\end{gathered}
$$

Remark 4. Error analysis with exponential decay kernel and Mittag-Leffler kernel can be obtained likewise. Therefore, we misplaced the error analysis for them.

\section{Conclusion}

In this paper, we investigated the fractional MHD incompressible couple stress fluid flow between two parallel plates. We discussed the discretization and the stability analysis for three different kernels. Additionally, we discussed the error analysis of the model in details.

\section{Data Availability}

No data were used to support this study.

\section{Conflicts of Interest}

The authors declare that they have no conflict of interest.

\section{References}

[1] T. Hayat, M. Khan, and M. Ayub, "Couette and Poiseuille flows of an Oldroyd 6-constant fluid with magnetic field," Journal of Mathematical Analysis and Applications, vol. 298, no. 1, pp. 225-244, 2004.

[2] T. Hayat, K. Hutter, S. Asghar, and A. M. Siddiqui, "MHD flows of an Oldroyd-B fluid," Mathematical and computer Modelling, vol. 36, no. 9-10, pp. 987-995, 2002.

[3] M. Kamran and I. Siddique, "MHD Couette and Poiseuille flow of a third grade fluid," Open Journal of Mathematical Analysis, vol. 1, no. 2, pp. 1-19, 2017.
[4] X. Si, L. Yuan, L. Zheng, Y. Shen, and L. Cao, "Lie group method for the modified model of MHD flow and heat transfer of a non-Newtonian fluid with prescribed heat flux over a moving porous plate," Journal of Molecular Liquids, vol. 220, pp. 768-777, 2016.

[5] Z. Shao, N. A. Shah, I. Tlili, U. Afzal, and M. S. Khan, "Hydromagnetic free convection flow of viscous fluid between vertical parallel plates with damped thermal and mass fluxes," Alexandria Engineering Journal, vol. 58, no. 3, pp. 989-1000, 2019.

[6] S. Nallapu, G. Radhakrishnacharya, and G. R. Kiran, "Effects of magnetic field and slip on a two-fluid model for couple stress fluid flow through a porous medium," International Journal of Pure and Applied Mathematics, vol. 113, no. 11, pp. 65-74, 2017.

[7] A. A. Kilbas, H. M. Srivastava, and J. J. Trujillo, Theory and Applications of Fractional Differential Equations, Elsevier, San Diego, 2006.

[8] I. Podlubny, Fractional differential equation, Academic Press, New York, 1999.

[9] M. Caputo and M. Fabrizio, "A new definition of fractional derivative without singular kernel," Prog. in Frac. Diff. and Appli., vol. 1, pp. 73-85, 2015.

[10] A. Atangana and D. Baleanu, "New fractional derivatives with nonlocal and non-singular kernel, theory and application to heat transfer model," Thermal Science, vol. 20, no. 2, pp. 763-769, 2016.

[11] K. S. Miller and B. Ross, An Introduction to the Fractional Calculus and Feactional Differential Equations, Willey, New York, 1993.

[12] M. U. Saleem, M. Farman, A. Ahmad, M. Naeem, and M. O. Ahmad, "Fractional order derivative glucose insulin model to control the type 1 diabetes mellitus," Punjab University Journal of Mathematics, vol. 51, no. 4, pp. 97-113, 2019.

[13] M. Farman, A. Akgül, A. Ahmad, and S. Imtiaz, "Analysis and dynamical behavior of fractional order cancer model with vaccine strategy," Mathematical Method in the Applied Sciences, 2020.

[14] B. Ghanbari, “A fractional system of delay differential equation with nonsingular kernels in modeling hand-foot-mouth disease," Advances in Difference Equations, vol. 2020, Article ID 536, 2020.

[15] B. Ghanbari and J. F. Gómez-Aguilar, "Two efficient numerical schemes for simulating dynamical systems and capturing chaotic behaviors with Mittag-Leffler memory," Engineering with Computers, 2020.

[16] G. Rahman, K. S. Nisar, B. Ghanbari, and T. Abdeljawad, “On generalized fractional integral inequalities for the monotone weighted Chebyshev functionals," Advances in Difference Equations, vol. 2020, no. 1, Article ID 368, 2020.

[17] B. Ghanbari and C. Cattani, "On fractional predator and prey models with mutualistic predation including non-local and nonsingular kernels," Chaos, Solitons \& Fractals, vol. 136, article 109823, 2020.

[18] K. Munusamy, C. Ravichandran, K. S. Nisar, and B. Ghanbari, "Existence of solutions for some functional integrodifferential equations with nonlocal conditions," Mathematical Methods in the Applied Sciences, vol. 43, no. 17, pp. 10319-10331, 2020.

[19] B. Ghanbari, H. Günerhan, and H. M. Srivastava, “An application of the Atangana-Baleanu fractional derivative in mathematical biology: a three-species predator-prey model," Chaos, Solitons \& Fractals, vol. 138, article 109910, 2020. 
[20] S. Djilali, B. Ghanbari, S. Bentout, and A. Mezouaghi, "TuringHopf bifurcation in a diffusive mussel-algae model with timefractional-order derivative," Chaos, Solitons \& Fractals, vol. 138, article 109954, 2020.

[21] I. Siddique, I. Tlili, S. M. Bukhari, and Y. Mahsud, "Heat transfer analysis in convective flows of fractional second grade fluids with Caputo-Fabrizio and Atangana-Baleanu derivative subject to Newtonion heating," Mechanics of Time-Dependent Materials, vol. 25, no. 3, pp. 291-311, 2021.

[22] W. Na, N. A. Shah, I. Tlili, and I. Siddique, "Maxwell fluid flow between vertical plates with damped shear and thermal flux: Free convection," Chinese Journal of Physics, vol. 65, pp. 367-376, 2020.

[23] A. Atangana, "Extension of rate of change concept: From local to nonlocal operators with applications," Results in Physics, vol. 19, article 103515, 2020.

[24] M. Farman, M. U. Saleem, A. Ahmad, and M. O. Ahmad, "Analysis and numerical solution of SEIR epidemic model of measles with non- integer time fractional derivatives by using Laplace Adomian decomposition method," Ain Shams Engineering Journal, vol. 9, no. 4, pp. 3391-3397, 2018.

[25] B. Ghanbari and S. Djilali, "Mathematical analysis of a fractional-order predator-prey model with prey social behavior and infection developed in predator population," Chaos, Solitons \& Fractals, vol. 138, article 109960, 2020.

[26] B. Ghanbari and A. Atangana, "Some new edge detecting techniques based on fractional derivatives with non-local and nonsingular kernels," Advances in Difference Equations, vol. 2020, no. 1, Article ID 435, 2020.

[27] A. Atangana, "Fractional discretization: the African's tortoise walk," Chaos, Solitons and Fractals, vol. 130, article 109399, 2020.

[28] A. Atangana, "Fractal-fractional differentiation and integration: connecting fractal calculus and fractional calculus to predict complex system," Chaos, Solitons and Fractals, vol. 102, pp. 396-406, 2017.

[29] A. Atangana and A. S. Qureshi, "Modeling attractors of chaotic dynamical systems with fractal-fractional operators," Chaos, Solitons and Fractals, vol. 123, pp. 320-337, 2019. 